\title{
Convexity Bounds for BSDE Solutions, With Applications to Indifference Valuation
}

\section{Journal Article}

\section{Author(s):}

Frei, Christoph; Malamud, Semyon; Schweizer, Martin

Publication date:

2011-06

Permanent link:

https://doi.org/10.3929/ethz-b-000019094

\section{Rights / license:}

In Copyright - Non-Commercial Use Permitted

Originally published in:

Probability Theory and Related Fields 150(1-2), https://doi.org/10.1007/s00440-010-0273-z 


\title{
Convexity bounds for BSDE solutions, with applications to indifference valuation
}

\author{
Christoph Frei · Semyon Malamud · Martin Schweizer
}

Received: 18 March 2009 / Revised: 10 January 2010 / Published online: 11 March 2010

(C) Springer-Verlag 2010

\begin{abstract}
We consider backward stochastic differential equations (BSDEs) with a particular quadratic generator and study the behaviour of their solutions when the probability measure is changed, the filtration is shrunk, or the underlying probability space is transformed. Our main results are upper bounds for the solutions of the original BSDEs in terms of solutions to other BSDEs which are easier to solve. We illustrate our results by applying them to exponential utility indifference valuation in a multidimensional Itô process setting.
\end{abstract}

Keywords Quadratic BSDE · Convexity bounds · Symmetrisation · Indifference valuation $\cdot$ Exponential utility

Mathematics Subject Classification (2000) $\quad 60 \mathrm{H} 10 \cdot 91 \mathrm{~B} 28 \cdot 60 \mathrm{G} 35$

\section{Introduction}

Backward stochastic differential equations (BSDEs) play an important role in mathematical finance; see El Karoui et al. [6] for an early overview. Existence and uniqueness results are well known both for Lipschitz and for quadratic drivers; see

\footnotetext{
C. Frei

CMAP, École Polytechnique, 91128 Palaiseau Cedex, France

e-mail: frei@cmap.polytechnique.fr

S. Malamud

Swiss Finance Institute, EPFL, 1015 Lausanne, Switzerland

e-mail: semyon.malamud@epfl.ch
}

M. Schweizer $(\bowtie)$

Department of Mathematics, ETH Zurich, 8092 Zurich, Switzerland

e-mail: martin.schweizer@math.ethz.ch 
Kobylanski [12]. In this paper, we study a particular class of quadratic BSDEs of the form

$$
\Gamma_{s}=G-\int_{s}^{T}\left(f\left(\Lambda_{r}, Z_{r}+\alpha_{r}\right)+\chi_{r}\right) \mathrm{d} r+\int_{s}^{T} Z_{r} \mathrm{~d} B_{r}, \quad 0 \leq s \leq T,
$$

where $f\left(\Lambda_{r}, Z_{r}+\alpha_{r}\right):=\frac{1}{2}\left(Z_{r}+\alpha_{r}\right)^{\prime} \Lambda_{r}^{-1}\left(Z_{r}+\alpha_{r}\right)$ and the processes $\chi, \alpha, \Lambda$ take values in $\mathbb{R}, \mathbb{R}^{n}$ and the set $\mathcal{S}^{n}$ of symmetric strictly positive definite matrices, respectively. Since there is no general formula for the solution $\Gamma$ of (1.1), we want to find bounds on $\Gamma$ that can be computed more explicitly. To that end, we first show that $f(A, z)$ is jointly convex, deduce that $\Gamma$ is jointly concave in $(G, \Lambda, \alpha, \chi)$, and then prove convexity bounds via three different routes, as follows.

In general, a BSDE is based on a probability space, a filtration and a probability measure. By changing in (1.1) each of these ingredients in a suitable way, we obtain other BSDEs whose solutions are upper bounds for $\Gamma$ due to concavity. Finding bounds for these changed BSDEs or solving them is easier than for the original (1.1), because they are driven by a lower-dimensional Brownian motion or, in some sense, their matrix-valued process $\Lambda$ is more regular.

We start by changing the probability measure. Our first main result, Theorem 3.1, characterises $\Gamma$ as the essential infimum and supremum of certain conditional expectations. In particular, it gives upper bounds for $\Gamma$, which depend on the maximal eigenvalue of $\Lambda$. This shows that $\Lambda$ is the crucial factor in finding good bounds, or even an explicit formula for $\Gamma$. The latter is easy if $\Lambda=c I$ for some constant $c$, and we prove in Corollary 3.2 that the converse holds as well. As a consequence, we then focus on improving the form of $\Lambda$ by projecting and/or symmetrising the BSDE (1.1).

For the projection, we change the filtration. The solution $\Gamma$ of (1.1) relates to the filtration $\mathbb{F}^{B}$ generated by $B=(\bar{B}, \underline{B})^{\prime}$, and our second main result, Theorem 3.3, gives an upper bound for $\Gamma$ in terms of the solution $\bar{\Gamma}$ to the BSDE $(\overline{1.1})$ obtained by projecting (1.1) onto $\mathbb{F}^{\bar{B}}$. The projected BSDE $(\overline{1.1})$ is in general easier to solve and the maximal eigenvalue of $\bar{\Lambda}$ is lower because the dimension $\bar{n}$ of $\bar{B}$ is smaller.

Finally, we change the probability space. We work on Wiener space and study how symmetrisation operations via orthogonal transformations there affect the BSDE (1.1). Our third main result, Theorem 3.7, gives an explicit upper bound for $\Gamma$ in terms of the symmetrised parameters $(G, \Lambda, \alpha, \chi)^{\mathrm{Sym}}$. The proof combines Theorem 3.1 with a result showing that, due to concavity, averaging the probability space over a set of orthogonal transformations increases the solution of (1.1).

The paper is structured as follows. We lay out preliminaries and prove the basic concavity property in Sect. 2.1. All our main results for the BSDE (1.1) have analogues in terms of solutions to partial differential equations (PDEs), which actually provided the original motivation and inspiration; see for instance Alvino et al. [1]. Section 2.2 discusses these connections in some more detail, and Sect. 3 contains the main results explained above. In Sect. 4 , we briefly recall the concept of exponential utility indifference valuation for a contingent claim $G$ in an incomplete financial market. It is well known that the corresponding dynamic value process $V^{G}$, or rather 
$\Gamma=-\frac{1}{\gamma} \log \left(-V^{G}\right)$, satisfies a quadratic BSDE; see for instance $\mathrm{Hu}$ et al. [9]. But since this BSDE is not of the form (1.1), we still have to do some work in Sect. 5 before we can apply our main results. We also discuss there in a concrete example why the symmetrisation techniques may, but need not lead to better bounds for $\Gamma$. Finally, the Appendix contains some proofs and auxiliary results.

\section{A quadratic convex BSDE}

This section serves as preparation for the main results. We first introduce notation and show some properties of quadratic BSDEs in Sect. 2.1, and then motivate in Sect. 2.2 the BSDE results of Sect. 3 by presenting their PDE analogues.

\subsection{Preliminaries}

We work on a finite time interval $[0, T]$ for a fixed $T>0$ and a filtered probability space $\left(\Omega, \mathcal{F}, \mathbb{F}=\left(\mathcal{F}_{s}\right)_{0 \leq s \leq T}, P\right)$, where $\mathcal{F}=\mathcal{F}_{T}$ and $\mathbb{F}$ is the augmented filtration generated by an $n$-dimensional Brownian motion $B$. Unless specified differently, all notions depending on a filtered probability space refer in Sects. 2 and 3 to $(\Omega, \mathcal{F}, \mathbb{F}, P)$, and $t \in[0, T]$ is fixed. For $(n \times n)$-matrices, we denote by $\mathcal{S}^{n}$ the set of symmetric strictly positive definite ones, by $\mathrm{GL}(n)$ and $\mathrm{O}(n)$ the invertible respectively orthogonal ones, and by $I$ the identity. For a diagonalisable matrix $A$, we write $\operatorname{spec}(A)$ for the spectrum (the set of eigenvalues) and $\operatorname{tr}(A)$ for the trace of $A$. We shall use several times that standard operations from linear algebra can be done in a measurable way. This includes eigenvalues, eigenvectors and diagonalisation; see Corollary 4 of Azoff [3]. Finally, we denote by $\mathcal{E}(N)_{s}:=\exp \left(N_{s}-\frac{1}{2}\langle N\rangle_{s}\right), 0 \leq s \leq T$, the stochastic exponential of a continuous semimartingale $N$.

Let us consider the BSDE

$$
\Gamma_{s}=G-\int_{s}^{T}\left(f\left(\Lambda_{r}, Z_{r}+\alpha_{r}\right)+\chi_{r}\right) \mathrm{d} r+\int_{s}^{T} Z_{r} \mathrm{~d} B_{r}, \quad 0 \leq s \leq T,
$$

where the function $f: \mathcal{S}^{n} \times \mathbb{R}^{n} \rightarrow \mathbb{R}$ is given by

$$
f(A, z):=\frac{1}{2} z^{\prime} A^{-1} z \text { for }(A, z) \in \mathcal{S}^{n} \times \mathbb{R}^{n} .
$$

The terminal value $G$ is (usually) in $L^{\infty}$, the process $\Lambda$ is $\mathcal{S}^{n}$-valued and predictable with eigenvalues uniformly bounded away from zero and infinity, and $\alpha, \chi$ are $\mathbb{R}^{n}$-, $\mathbb{R}$-valued uniformly bounded predictable processes. A (generalised) solution of (2.1) is a pair $(\Gamma, Z)$ satisfying (2.1), where $\Gamma$ is a real-valued (not necessarily) bounded continuous semimartingale and $Z$ is an $\mathbb{R}^{n}$-valued predictable process with $\int_{0}^{T}\left|Z_{s}\right|^{2} \mathrm{~d} s<\infty$ almost surely. To emphasise the dependence on $G, \Lambda, \alpha$ and $\chi$, we write $(\Gamma(G, \Lambda, \alpha, \chi), Z(G, \Lambda, \alpha, \chi))$ for a solution of (2.1), and we sometimes call $\Gamma(G, \Lambda, \alpha, \chi)$ alone a solution of (2.1). 
Remark 2.1 For ease of exposition, we formulate and prove all our results for bounded data $G, \Lambda, \alpha, \chi$. Extensions to unbounded settings with exponential moment conditions are partly possible; this is discussed in more detail in Remark 3.8.

Lemma 2.2 There exists a unique solution $(\Gamma, Z)$ of $(2.1)$, and $\int Z \mathrm{~d} B$ is a BMOmartingale.

Proof Existence follows from Theorem 2.3 of Kobylanski [12], and uniqueness and $B M O$-property from Proposition 7 and Theorem 8 of Mania and Schweizer [13].

In Lemma A. 1 in the Appendix, we show that $f$ is jointly convex. This is the basis for the following result.

Proposition 2.3 The solution $\Gamma(G, \Lambda, \alpha, \chi)$ of $(2.1)$ is jointlyconcave in $(G, \Lambda, \alpha, \chi)$.

Remark 2.4 It is BSDE folklore that convexity of the generator implies (under some assumptions) that the solution is concave; see for instance Proposition 3.5 of El Karoui et al. [6], where the generator is fairly general, but must satisfy a Lipschitz condition in $Z_{r}$ and in $\Gamma_{r}$. We need the variant in Proposition 2.3 with a specific quadratic generator for our later results.

Proof of Proposition 2.3 Let $\mu \in[0,1], G^{i} \in L^{\infty}$, let $\Lambda^{i}$ be predictable $\mathcal{S}^{n}$-valued with eigenvalues bounded away from zero and infinity and let bounded predictable $\alpha^{i}$ be $\mathbb{R}^{n}$-valued and $\chi^{i}$ be $\mathbb{R}$-valued, $i=1,2$. We set $G^{3}:=\mu G^{1}+(1-\mu) G^{2}$, define $\Lambda^{3}, \alpha^{3}, \chi^{3}$ analogously and denote by $\left(\Gamma^{i}, Z^{i}\right), i=1,2,3$, the solutions of (2.1) corresponding to $\left(G^{i}, \Lambda^{i}, \alpha^{i}, \chi^{i}\right)$. By Lemma 2.2, each of these is unique and $\int Z^{i} \mathrm{~d} B$ are $B M O$-martingales. Since $\mu \Gamma_{T}^{1}+(1-\mu) \Gamma_{T}^{2}=\mu G^{1}+(1-\mu) G^{2}=G^{3},(2.1)$ and Lemma A.1 yield

$$
\begin{aligned}
\Gamma_{s}^{3}- & \left(\mu \Gamma_{s}^{1}+(1-\mu) \Gamma_{s}^{2}\right) \\
= & \int_{s}^{T}\left(\mu f\left(\Lambda_{r}^{1}, Z_{r}^{1}+\alpha_{r}^{1}\right)+(1-\mu) f\left(\Lambda_{r}^{2}, Z_{r}^{2}+\alpha_{r}^{2}\right)-f\left(\Lambda_{r}^{3}, Z_{r}^{3}+\alpha_{r}^{3}\right)\right) \mathrm{d} r \\
& -\int_{s}^{T}\left(\mu Z_{r}^{1}+(1-\mu) Z_{r}^{2}-Z_{r}^{3}\right) \mathrm{d} B_{r} \\
\geq & \int_{s}^{T}\left(f\left(\Lambda_{r}^{3}, \mu Z_{r}^{1}+(1-\mu) Z_{r}^{2}+\alpha_{r}^{3}\right)-f\left(\Lambda_{r}^{3}, Z_{r}^{3}+\alpha_{r}^{3}\right)\right) \mathrm{d} r \\
& -\int_{s}^{T}\left(\mu Z_{r}^{1}+(1-\mu) Z_{r}^{2}-Z_{r}^{3}\right) \mathrm{d} B_{r} \quad 0 \leq s \leq T \\
= & \left.-\int_{s}^{T}\left(\mu Z_{r}^{1}+(1-\mu) Z_{r}^{2}-Z_{r}^{3}\right)\left(\mathrm{d} B_{r}-\kappa_{r} \mathrm{~d} r\right), \quad 0.3\right)
\end{aligned}
$$


with $\kappa:=\frac{1}{2}\left(\Lambda^{3}\right)^{-1}\left(\mu Z^{1}+(1-\mu) Z^{2}+Z^{3}+2 \alpha^{3}\right)$. Since the eigenvalues of $\Lambda^{3}$ are bounded away from zero and $\alpha^{3}$ is bounded, $\int \kappa \mathrm{d} B$ is a $B M O$-martingale. By Theorem 3.6 of Kazamaki [11] and the $B M O(P)$-property of $\int Z^{i} \mathrm{~d} B$, the process $\int\left(\mu Z^{1}+(1-\mu) Z^{2}-Z^{3}\right)(\mathrm{d} B-\kappa \mathrm{d} r)$ is thus also a $B M O(\tilde{P})$-martingale for the probability measure $\tilde{P}$ given by $\frac{\mathrm{d} \tilde{P}}{\mathrm{~d} P}:=\mathcal{E}\left(\int \kappa \mathrm{d} B\right)_{T}$. Taking $\left(\tilde{P}, \mathcal{F}_{S}\right)$-conditional expectations in (2.3) yields $\Gamma_{s}^{3}-\left(\mu \Gamma_{s}^{1}+(1-\mu) \Gamma_{s}^{2}\right) \geq 0$ for any $s \in[0, T]$, which concludes the proof since the $\Gamma^{i}$ are continuous.

The basic and well-known case is when $\alpha \equiv 0, \chi \equiv 0$ and $\Lambda=c I$ for a fixed $c>0$. The BSDE (2.1) then simplifies to

$$
\Gamma_{s}=G-\int_{s}^{T} \frac{1}{2 c}\left|Z_{r}\right|^{2} \mathrm{~d} r+\int_{s}^{T} Z_{r} \mathrm{~d} B_{r}=\Gamma_{0}-c \log \mathcal{E}\left(\int \frac{1}{c} Z \mathrm{~d} B\right)_{s}, \quad 0 \leq s \leq T .
$$

Due to Itô's formula, its explicit solution is

$$
\Gamma_{s}=-c \log E\left[\exp (-G / c) \mid \mathcal{F}_{s}\right], \quad 0 \leq s \leq T
$$

because $\int Z \mathrm{~d} B$ is a $B M O$-martingale by Lemma 2.2, and hence $\mathcal{E}\left(\int \frac{1}{c} Z \mathrm{~d} B\right)$ is a martingale by Theorem 2.3 of Kazamaki [11].

\subsection{Motivation for the convexity results}

Before we state and prove in Sect. 3 convexity results for the solution of the BSDE (2.1), we explain the basic ideas using PDEs. Since we only want to provide motivation, we look at the results exclusively for time 0 and ignore here all technical issues like existence of smooth solutions, interchanging expectation and differential, etc.

Assume in (2.1) that $\alpha, \chi$ and $\Lambda$ are all deterministic and $G=g\left(B_{T}\right)$ for a smooth function $g: \mathbb{R} \rightarrow \mathbb{R}$. In this Markovian setting, one can derive from Itô's formula that the solution $(\Gamma, Z)$ of $(2.1)$ satisfies

$$
\Gamma_{s}=u\left(s, B_{s}\right), \quad Z_{s}=-\nabla_{x} u\left(s, B_{s}\right) \text { for } s \in[0, T],
$$

where $u:[0, T] \times \mathbb{R}^{n} \rightarrow \mathbb{R}$ solves the PDE

$$
\left.\begin{array}{l}
\frac{\partial}{\partial s} u(s, x)+\frac{1}{2} \Delta_{x} u(s, x)-f\left(\Lambda(s), \alpha(s)-\nabla_{x} u(s, x)\right)-\chi(s)=0, \\
u(T, x)=g(x) \text { for } s \in[0, T) \text { and } x \in \mathbb{R}^{n} .
\end{array}\right\}
$$

Each of our three main results yields an upper bound for $\Gamma$. We look in the following as illustration at the PDE analogue of the symmetrisation result in Theorem 3.7. The other BSDE theorems have similar PDE analogues. For ease of notation, we take $\alpha$, $\chi, \Lambda$ all constant. 
Symmetrisation inequalities play an important role in the theory of linear parabolic PDEs; see e.g. Alvino et al. [1] and the references therein. They show that in some sense, the solution of a symmetrised PDE dominates the symmetrised solution of the original PDE. Theorem 3.7 below can be viewed as an analogue of these results for nonlinear parabolic PDEs. To explain the connection, let Perm $\subseteq \mathrm{O}(n)$ be the group of permutations of length $n$, where we identify permutations with orthogonal matrices. We define

$$
\Lambda^{\mathrm{Sym}}=\frac{1}{n !} \sum_{O \in \operatorname{Perm}} O^{\prime} \Lambda O, \quad \alpha^{\mathrm{Sym}}:=\frac{1}{n !} \sum_{O \in \operatorname{Perm}} O^{\prime} \alpha, g^{\mathrm{Sym}}:=\frac{1}{n !} \sum_{O \in \operatorname{Perm}}(g \circ O) .
$$

Let $\tilde{u}:[0, T] \times \mathbb{R}^{n} \rightarrow \mathbb{R}$ solve the symmetrised PDE

$$
\left.\begin{array}{l}
\frac{\partial}{\partial s} \tilde{u}(s, x)+\frac{1}{2} \Delta_{x} \tilde{u}(s, x)-f\left(\Lambda^{\operatorname{Sym}}, \alpha^{\operatorname{Sym}}-\nabla_{x} \tilde{u}(s, x)\right)-\chi=0, \\
\tilde{u}(T, x)=g^{\operatorname{Sym}}(x) \text { for } s \in[0, T) \text { and } x \in \mathbb{R}^{n} .
\end{array}\right\}
$$

Then Proposition 3.6 below tells us that

$$
\tilde{u}(0,0) \geq u(0,0)
$$

We justify this here by a PDE comparison argument. For $O \in$ Perm, we have $\left.\nabla_{y} u(s, y)\right|_{y=O x}=O \nabla_{x} u(s, O x),\left.\Delta_{y} u(s, y)\right|_{y=O x}=\Delta_{x} u(s, O x)$ and, from (2.2),

$$
f\left(\Lambda, \alpha-O \nabla_{x} u(s, O x)\right)=f\left(O^{\prime} \Lambda O, O^{\prime} \alpha-\nabla_{x} u(s, O x)\right)
$$

Due to (2.5), the symmetrised function $\bar{u}(s, x):=\frac{1}{n !} \sum_{O \in \operatorname{Perm}} u(s, O x)$ solves

$$
\begin{aligned}
& \frac{\partial}{\partial s} \bar{u}(s, x)+\frac{1}{2} \Delta_{x} \bar{u}(s, x)-\frac{1}{n !} \sum_{O \in \operatorname{Perm}} f\left(O^{\prime} \Lambda O, O^{\prime} \alpha-\nabla_{x} u(s, O x)\right)-\chi=0 \\
& \bar{u}(T, x)=g^{\operatorname{Sym}}(x) \text { for } s \in[0, T) \text { and } x \in \mathbb{R}^{n}
\end{aligned}
$$

By Lemma A.1, $f$ is jointly convex, which yields

$$
\frac{1}{n !} \sum_{O \in \operatorname{Perm}} f\left(O^{\prime} \Lambda O, O^{\prime} \alpha-\nabla_{x} u(s, O x)\right) \geq f\left(\Lambda^{\mathrm{Sym}}, \alpha^{\mathrm{Sym}}-\nabla_{x} \bar{u}(s, x)\right) .
$$

Since $\bar{u}(0,0)=u(0,0)$, we obtain (2.7) by comparing (2.6) and (2.8). Now fix $c>0$. One can check that the solution $\hat{u}$ of

$$
\left.\begin{array}{l}
\frac{\partial}{\partial s} \hat{u}(s, x)+\frac{1}{2} \Delta_{x} \hat{u}(s, x)-\frac{1}{2 c}\left|\alpha^{\operatorname{Sym}}-\nabla_{x} \hat{u}(s, x)\right|^{2}-\chi=0, \\
\hat{u}(T, x)=g^{\operatorname{Sym}}(x) \text { for } s \in[0, T) \text { and } x \in \mathbb{R}^{n}
\end{array}\right\}
$$


satisfies

$$
\hat{u}(0,0)=-c \log E\left[\exp \left(-g^{\operatorname{Sym}}\left(B_{T}\right)+\int_{0}^{T} \alpha^{\operatorname{Sym}} \mathrm{d} B_{s}\right)^{\frac{1}{c}}\right]-\int_{0}^{T} \chi \mathrm{d} s .
$$

To compare (2.6) with (2.9), we assume that $\Lambda=\operatorname{diag}\left(\Lambda^{11}, \ldots, \Lambda^{n n}\right)$ is of diagonal form and set $c:=\sup _{s \in[0, T]} \frac{1}{n} \operatorname{tr}\left(\Lambda_{s}\right)$ (if $\Lambda$ is time-dependent). Then $\Lambda^{\operatorname{Sym}}=\frac{1}{n} \operatorname{tr}(\Lambda) I$ since $\Lambda$ is diagonal, and hence

$$
f\left(\Lambda^{\mathrm{Sym}}, \alpha^{\mathrm{Sym}}-x\right) \geq \frac{1}{2 c}\left|\alpha^{\mathrm{Sym}}-x\right|^{2} \quad \text { for } x \in \mathbb{R}^{n}
$$

We thus expect by comparing (2.6) and (2.9) that $\tilde{u}(0,0) \leq \hat{u}(0,0)$, which gives via (2.7) and (2.10) an explicit upper bound for the solution of the original PDE (2.5). Theorem 3.7 makes this statement precise and provides a proof in a general BSDE setting.

\section{Convexity results for quadratic BSDEs}

This section contains our three main results. We study how the solution of the BSDE (2.1) is affected if we change the probability measure, shrink the filtration, or symmetrise the probability space.

\subsection{Changing the probability measure}

For any predictable $\kappa$ such that $\int \kappa \mathrm{d} B$ is a $B M O$-martingale, we define

$$
\frac{\mathrm{d} P^{\kappa}}{\mathrm{d} P}:=\mathcal{E}\left(-\int \kappa \mathrm{d} B\right)_{T}, \quad B^{\kappa}:=B+\int \kappa \mathrm{d} s
$$

and note that $B^{\kappa}$ is a Brownian motion under the probability measure $P^{\kappa}$. Recalling that $t \in[0, T]$ is fixed and spec denotes the spectrum, we define

$$
\begin{aligned}
\delta_{t}^{\max } & :=\sup _{s \in[t, T]}\left\|\max \operatorname{spec}\left(\Lambda_{S}\right)\right\|_{L^{\infty}}, \quad \delta_{t}^{\min }:=\inf _{s \in[t, T]} \frac{1}{\left\|\max \operatorname{spec}\left(\Lambda_{s}^{-1}\right)\right\|_{L^{\infty}}}, \\
G_{t}^{\kappa} & :=G-\int_{t}^{T}\left(\chi_{s}+\frac{1}{2} \kappa_{s}^{\prime} \Lambda_{s} \kappa_{s}\right) \mathrm{d} s-\int_{t}^{T}\left(\alpha_{s}+\Lambda_{s} \kappa_{S}\right) \mathrm{d} B_{s} .
\end{aligned}
$$

For $\delta>0$, let $\mathcal{K}^{\delta}$ be the set of all predictable $\mathbb{R}^{n}$-valued processes $\kappa$ such that $\int \kappa \mathrm{d} B$ is in $B M O$ and there exist $p>1$ and a constant $C$ such that 


$$
\begin{aligned}
& E_{P^{\kappa}}\left[\exp \left(\int_{t}^{T} \frac{1}{2} \kappa_{s}^{\prime} \Lambda_{s} \kappa_{s} \mathrm{~d} s+\int_{t}^{T} \Lambda_{s} \kappa_{s} \mathrm{~d} B_{s}\right)^{p / \delta} \mid \mathcal{F}_{\tau}\right] \\
& \left.\leq C E_{P^{\kappa}}\left[\exp \left(\int_{t}^{T} \frac{1}{2} \kappa_{s}^{\prime} \Lambda_{s} \kappa_{S} \mathrm{~d} s+\int_{t}^{T} \Lambda_{s} \kappa_{s} \mathrm{~d} B_{s}\right)^{1 / \delta}\right] \mathcal{F}_{\tau}\right]^{p}<\infty,
\end{aligned}
$$

for any stopping time $\tau$ with values in $[t, T]$. The latter condition says that the martingale

$$
E_{P^{\kappa}}\left[\exp \left(\int_{t}^{T} \frac{1}{2} \kappa_{s}^{\prime} \Lambda_{s} \kappa_{s} \mathrm{~d} s+\int_{t}^{T} \Lambda_{s} \kappa_{S} \mathrm{~d} B_{s}\right)^{1 / \delta} \mid \mathcal{F}_{r}\right], \quad t \leq r \leq T,
$$

satisfies the reverse Hölder inequality $R_{p}\left(P^{\kappa}\right)$. Each $\mathcal{K}^{\delta}$ contains all bounded predictable processes and also some unbounded processes. In fact, for any predictable $\kappa$ with $\int \kappa \mathrm{d} B$ in $B M O$, there exists a constant $c>0$ such that $c \kappa \in \mathcal{K}^{\delta}$. This follows from Lemma A. 2 in the Appendix, which shows that a predictable process $\kappa$ is in $\mathcal{K}^{\delta}$ if the $B M O$-norm of $\int \kappa \mathrm{d} B$ is small enough. Furthermore, (3.3) is equivalent to

$$
E_{P^{\kappa}}\left[\exp \left(-G_{t}^{\kappa}\right)^{p / \delta} \mid \mathcal{F}_{\tau}\right] \leq C E_{P^{\kappa}}\left[\exp \left(-G_{t}^{\kappa}\right)^{1 / \delta} \mid \mathcal{F}_{\tau}\right]^{p}<\infty
$$

since $G, \chi$ and $\alpha$ are bounded. We set $\mathcal{K}:=\mathcal{K}_{t}^{\delta_{t}^{\max }} \cap \mathcal{K}_{t}^{\delta_{t}^{\min }}$.

Theorem 3.1 The solution $\Gamma$ of the BSDE (2.1) satisfies

$$
\begin{aligned}
\Gamma_{t} & =-\underset{\kappa \in \mathcal{K}}{\operatorname{ess} \sup } \log E_{P^{\kappa}}\left[\exp \left(-G_{t}^{\kappa} / \delta_{t}^{\max }\right) \mid \mathcal{F}_{t}\right]^{\delta_{t}^{\max }} \\
& =-\underset{\kappa \in \mathcal{K}}{\operatorname{essinf}} \log E_{P^{\kappa}}\left[\exp \left(-G_{t}^{\kappa} / \delta_{t}^{\min }\right) \mid \mathcal{F}_{t}\right]^{\delta_{t}^{\min }},
\end{aligned}
$$

and for every $\kappa \in \mathcal{K}$, there exists an $\mathcal{F}_{t}$-measurable random variable $\delta_{t}^{\kappa, G}$ with values in $\left[\delta_{t}^{\min }, \delta_{t}^{\max }\right]$ such that

$$
\Gamma_{t}=-\left.\log E_{P^{\kappa}}\left[\exp \left(-G_{t}^{\kappa} / \delta\right) \mid \mathcal{F}_{t}\right]^{\delta}\right|_{\delta=\delta_{t}^{\kappa, G}}
$$

Theorem 3.1 illustrates the importance of the process $\Lambda$ in the BSDE (2.1). Indeed, $\Lambda$ determines via (3.2) the eigenvalue bounds $\delta_{t}^{\min , \max }$ and hence the range of $\delta_{t}^{\kappa, G}$ in (3.7). If $\Lambda=c I$ for a constant $c$, we have $\delta_{t}^{\min }=\delta_{t}^{\max }=c=\delta_{t}^{\kappa, G}$, and (3.7) is an explicit formula for $\Gamma_{t}$ as distorted conditional expectation under $P^{\kappa}$. Corollary 3.2 below gives a converse: If for any $G$, the solution $\Gamma_{t}$ of the BSDE (2.1) is the distorted conditional expectation under some $P^{\kappa}$, then $\Lambda=c I$ for a constant $c$. Theorem 3.1 also generalises Theorem 2 of Frei and Schweizer [7], as we explain in Sect. 5.3. 
Moreover, we can recover the bound in Proposition 2.1 of Kobylanski [12] applied to the BSDE (2.1); indeed, for $\kappa=-\Lambda^{-1} \alpha \in \mathcal{K},(3.5)$ yields

$$
\begin{aligned}
\Gamma_{t} & \leq-\log E_{P^{\kappa}}\left[\exp \left(-G+\int_{t}^{T}\left(\chi_{s}+\frac{1}{2} \alpha_{s}^{\prime} \Lambda_{s}^{-1} \alpha_{s}\right) \mathrm{d} s\right)^{1 / \delta_{t}^{\max } \mid} \mathcal{F}_{t}\right]^{\delta_{t}^{\max }} \\
& \leq-\log E_{P^{\kappa}}\left[\exp \left(-\left\|G^{+}\right\|_{L^{\infty}}-\int_{t}^{T}\left\|\chi_{s}+\frac{1}{2} \alpha_{s}^{\prime} \Lambda_{s}^{-1} \alpha_{s}\right\|_{L^{\infty}} \mathrm{d} s\right)^{1 / \delta_{t}^{\max } \mid} \mathcal{F}_{t}\right]^{\delta_{t}^{\max }} \\
& \leq\left\|G^{+}\right\|_{L^{\infty}}+\int_{t}^{T}\left\|\left|\chi_{s}\right|+\alpha_{s}^{\prime} \Lambda_{s}^{-1} \alpha_{s}\right\|_{L^{\infty}} \mathrm{d} s,
\end{aligned}
$$

which one can also derive from Proposition 2.1 of Kobylanski [12].

A result similar to (3.5) in Theorem 3.1 is Theorem 3.25 of Barrieu and El Karoui [4]. While their result holds for BSDEs with a more general convex generator, our procedure works only for BSDEs with a particular quadratic generator, but gives a better upper bound for the solution $\Gamma_{t}$ by fixing $\kappa \in \mathcal{K}$. The proof of Theorem 3.25 of [4] is based on the idea that a convex generator can be bounded from below independently of $Z$ by using the convex conjugate. In our case, we can find for the specific generator a better bound given by the generator of another quadratic BSDE which has an explicit solution. Moreover, we exploit the form of the generator to obtain also (3.6) so that we have both upper and lower bounds for $\Gamma_{t}$ by fixing $\kappa \in \mathcal{K}$.

From (3.5) we obtain upper bounds for $\Gamma_{t}$, which depend on the maximal eigenvalue of $\Lambda$. Our other two main results, Theorems 3.3 and 3.7, can be viewed as approaches to get better bounds by reducing $\delta_{t}^{\max }$ (and also changing $G$ ). In Theorem 3.3, we reduce the dimension $n$ of the BSDE by projecting it onto the filtration of a lower-dimensional Brownian motion, and replacing $\Lambda$ by its projection in principle lowers the maximal eigenvalue. Similarly, the symmetrisation in Theorem 3.7 makes the eigenvalues more similar and in particular reduces the maximal eigenvalue.

Proof of Theorem 3.1 We first show

$$
\Gamma_{t} \leq-\log E_{P^{\kappa}}\left[\exp \left(-G_{t}^{\kappa} / \delta_{t}^{\max }\right) \mid \mathcal{F}_{t}\right]^{\delta_{t}^{\max }}
$$

for any $\kappa \in \mathcal{K}$. We obtain from (2.1), (2.2) and (3.1) that

$$
\begin{aligned}
\Gamma_{s}= & G-\int_{s}^{T} \frac{1}{2}\left(Z_{r}+\alpha_{r}+\Lambda_{r} \kappa_{r}\right)^{\prime} \Lambda_{r}^{-1}\left(Z_{r}+\alpha_{r}+\Lambda_{r} \kappa_{r}\right) \mathrm{d} r \\
& -\int_{s}^{T}\left(\chi_{r}-\kappa_{r}^{\prime} \alpha_{r}-\frac{1}{2} \kappa_{r}^{\prime} \Lambda_{r} \kappa_{r}\right) \mathrm{d} r+\int_{s}^{T} Z_{r} \mathrm{~d} B_{r}^{\kappa}, \quad 0 \leq s \leq T .
\end{aligned}
$$


Define

$$
\begin{aligned}
\Gamma_{s}^{\kappa}:= & -\log E_{P^{\kappa}}\left[\exp \left(-G_{t}^{\kappa} / \delta_{t}^{\max }\right) \mid \mathcal{F}_{s}\right]_{t}^{\delta_{t}^{\max }} \\
& +\int_{t}^{s}\left(\chi_{r}+\frac{1}{2} \kappa_{r}^{\prime} \Lambda_{r} \kappa_{r}\right) \mathrm{d} r+\int_{t}^{s}\left(\alpha_{r}+\Lambda_{r} \kappa_{r}\right) \mathrm{d} B_{r}, \quad t \leq s \leq T .
\end{aligned}
$$

Using Itô's representation theorem as in Lemma 1.6.7 of Karatzas and Shreve [10] gives

$$
E_{P^{\kappa}}\left[\exp \left(-G_{t}^{\kappa} / \delta_{t}^{\max }\right) \mid \mathcal{F} .\right]=c^{\kappa} \mathcal{E}\left(\int Z^{\kappa} \mathrm{d} B^{\kappa}\right)
$$

for a constant $c^{\kappa}$ and a predictable $\mathbb{R}^{n}$-valued $Z^{\kappa}$ such that $\mathcal{E}\left(\int Z^{\kappa} \mathrm{d} B^{\kappa}\right)$ is a $P^{\kappa}$ martingale. Since $G, \chi$ and $\alpha$ are bounded, (3.2) and (3.3) imply that $\mathcal{E}\left(\int Z^{\kappa} \mathrm{d} B^{\kappa}\right)$ satisfies the reverse Hölder inequality $R_{p}\left(P^{\kappa}\right)$ for some $p>1$. Hence $\int Z^{\kappa} \mathrm{d} B^{\kappa}$ is in $B M O\left(P^{\kappa}\right)$ by Theorem 3.4 of Kazamaki [11], and so is $\int \bar{Z}^{\kappa} \mathrm{d} B^{\kappa}$ for

$$
\bar{Z}^{\kappa}:=\delta_{t}^{\max } Z^{\kappa}-\alpha-\Lambda \kappa
$$

A calculation based on (3.10) and (3.11) gives for $t \leq s \leq T$

$$
\begin{aligned}
\Gamma_{s}^{\kappa}= & G-\int_{s}^{T} \frac{1}{2 \delta_{t}^{\max }}\left|\bar{Z}_{r}^{\kappa}+\alpha_{r}+\Lambda_{r} \kappa_{r}\right|^{2} \mathrm{~d} r \\
& -\int_{s}^{T}\left(\chi_{r}-\kappa_{r}^{\prime} \alpha_{r}-\frac{1}{2} \kappa_{r}^{\prime} \Lambda_{r} \kappa_{r}\right) \mathrm{d} r+\int_{s}^{T} \bar{Z}_{r}^{\kappa} \mathrm{d} B_{r}^{\kappa},
\end{aligned}
$$

and comparing (3.13) and (3.9) yields similarly as in Proposition 2.3 that $\Gamma_{t} \leq \Gamma_{t}^{\kappa}$. This is (3.8).

Now set $\hat{\kappa}:=-\Lambda^{-1}(Z+\alpha)$ with $Z$ from (3.9). Then $\int \hat{\kappa} \mathrm{d} B \in B M O$ since $\alpha$ is bounded, $\int Z \mathrm{~d} B \in B M O$ and $\Lambda^{-1}$ is bounded. Moreover, $G_{t}^{\hat{\kappa}}$ is $\mathcal{F}_{t}$-measurable; hence $\hat{\kappa}$ satisfies (3.4) and thus (3.3) for any $\delta>0$, and so $\hat{\kappa}$ is in $\mathcal{K}$. Again using that $G_{t}^{\hat{\kappa}}$ is $\mathcal{F}_{t}$-measurable plus (3.9) and (3.2) shows that

$$
\Gamma_{t}=G_{t}^{\hat{\kappa}}=-\log E_{P^{\hat{\kappa}}}\left[\exp \left(-G_{t}^{\hat{\kappa}} / \delta_{t}^{\max }\right) \mid \mathcal{F}_{t}\right]^{\delta_{t}^{\max }} .
$$

Hence we have (3.5), and (3.6) is proved analogously. (3.7) now follows by the same interpolation argument as in Theorem 1 of Frei and Schweizer [7]; the required $P^{\kappa}$-integrable majorant for the family $\left\{\exp \left(-G_{t}^{\kappa} / \delta\right) \mid \delta \in\left[\delta_{t}^{\min }, \delta_{t}^{\max }\right]\right\}$ is $\exp \left(-G_{t}^{\kappa} / \delta_{t}^{\min }\right)+1$.

We next study when $\Gamma_{t}$ from (2.1) is a distorted conditional expectation under some $P^{\kappa}$. For $\delta>0$ and $\kappa \in \mathcal{K}^{\delta}$, let $L^{\delta, \kappa}$ be the set of random variables $G$ such that $G_{t}^{\kappa}$ 
from (3.2) satisfies the reverse Hölder inequality (3.4) for some $p>1$. The definition of $\mathcal{K}^{\delta}$ implies that $L^{\infty} \subseteq L^{\delta, \kappa}$, but $G \in L^{\delta, \kappa}$ need not be bounded.

Corollary 3.2 The following are equivalent:

(a) There exists a constant $c>0$ such that

$$
\Lambda=c I \text { on } \rrbracket t, T \rrbracket(P \otimes \text { Leb }) \text {-a.e. }
$$

(b) There exists a constant $\delta \in\left[\delta_{t}^{\min }, \delta_{t}^{\max }\right]$ such that for all $\kappa \in \mathcal{K}^{\delta}$ and $G \in L^{\delta, \kappa}$, there exists a generalised solution $(\Gamma, Z)$ on $\llbracket t, T \rrbracket$ of $(2.1)$ such that $\int Z \mathrm{~d} B$ is a $B M O(P)$-martingale and

$$
\Gamma_{t}=-\log E_{P^{\kappa}}\left[\exp \left(-G_{t}^{\kappa} / \delta\right) \mid \mathcal{F}_{t}\right]^{\delta} .
$$

(c) There exist a constant $\delta \in\left[\delta_{t}^{\min }, \delta_{t}^{\max }\right]$ and a process $\kappa \in \mathcal{K}^{\delta}$ such that for all $G \in L^{\delta, \kappa}$, there exists a generalised solution $(\Gamma, Z)$ on $\llbracket t, T \rrbracket$ of $(2.1)$ such that $\int Z \mathrm{~d} B$ is a $B M O(P)$-martingale and (3.15) holds.

In this case, $c=\delta$.

Proof “(b) $\Longrightarrow$ (c)" is clear. To show "(a) $\Longrightarrow$ (b)", we use a similar argument as for Theorem 3.1. Take $\kappa \in \mathcal{K}$ and define $\Gamma^{\kappa}$ and $\bar{Z}^{\kappa}$ by (3.10) and (3.12) with $\delta_{t}^{\max }$ replaced by $\delta:=c$. Then $\int \bar{Z}^{\kappa} \mathrm{d} B^{\kappa}$ is again in $B M O\left(P^{\kappa}\right)$ so that $\int \bar{Z}^{\kappa} \mathrm{d} B$ is in $B M O(P)$, and like (3.13), we get

$$
\begin{aligned}
\Gamma_{s}^{\kappa}= & \Gamma_{t}^{\kappa}+\int_{t}^{s} \frac{1}{2 \delta}\left|\bar{Z}_{r}^{\kappa}+\alpha_{r}+\Lambda_{r} \kappa_{r}\right|^{2} \mathrm{~d} r \\
& +\int_{t}^{s}\left(\chi_{r}-\kappa_{r}^{\prime} \alpha_{r}-\frac{1}{2} \kappa_{r}^{\prime} \Lambda_{r} \kappa_{r}\right) \mathrm{d} r-\int_{t}^{s} \bar{Z}_{r}^{\kappa} \mathrm{d} B_{r}^{\kappa}, \quad t \leq s \leq T
\end{aligned}
$$

Plugging in (3.14) with $\delta=c$ shows after some computation that $\left(\Gamma^{\kappa}, \bar{Z}^{\kappa}\right)$ satisfies (2.1) on $\llbracket t, T \rrbracket$. Finally, (3.15) holds for $\Gamma:=\Gamma^{\kappa}$ by construction.

To prove " $(\mathrm{c}) \Longrightarrow(\mathrm{a})$ ", we define the predictable set

$$
\Upsilon_{1}:=\left\{(\omega, s) \in \rrbracket t, T \rrbracket \mid \min \operatorname{spec}\left(\Lambda_{s}(\omega)\right)<\delta\right\}
$$

and choose a predictable $\mathbb{R}^{n}$-valued process $v$ such that $\Lambda v=(\min \operatorname{spec}(\Lambda)) v$ and $|v|=1$ on $\rrbracket t, T \rrbracket$; so $v_{S}(\omega)$ is an eigenvector for the smallest eigenvalue of $\Lambda_{S}(\omega)$. Set

$$
\begin{aligned}
G:= & \int_{t}^{T}\left(\chi_{s}+\frac{1}{2} \kappa_{s}^{\prime} \Lambda_{s} \kappa_{s}\right) \mathrm{d} s+\int_{t}^{T}\left(\alpha_{s}+\Lambda_{s} \kappa_{s}\right) \mathrm{d} B_{s} \\
& -\int_{t}^{T} \mathbb{1}_{\Upsilon_{1}}(s) v_{s} \mathrm{~d} B_{s}+\int_{t}^{T} \mathbb{1}_{\Upsilon_{1}}(s)\left(\frac{1}{2 \delta}-v_{s}^{\prime} \kappa_{s}\right) \mathrm{d} s
\end{aligned}
$$


so that the corresponding $G_{t}^{\kappa}$ given by (3.2) satisfies

$$
\exp \left(-G_{t}^{\kappa} / \delta\right)=\mathcal{E}\left(\int \frac{1}{\delta} \mathbb{1}_{\Upsilon_{1}} v \mathrm{~d} B^{\kappa}\right)_{T} .
$$

Hence $G$ is in $L^{\delta, \kappa}$ by Theorem 3.4 of Kazamaki [11]; in fact, $\int \frac{1}{\delta} \mathbb{1}_{\Upsilon_{1}} v \mathrm{~d} B^{\kappa}$ is a $B M O\left(P^{\kappa}\right)$-martingale because its integrand is bounded.

Now (3.15), (3.16) and Itô's formula, (3.2) and (3.1) give with some calculations

$$
\begin{aligned}
\Gamma_{t}= & -\log E_{P^{\kappa}}\left[\exp \left(-G_{t}^{\kappa} / \delta\right) \mid \mathcal{F}_{t}\right]^{\delta} \\
= & G-\int_{t}^{T}\left(\chi_{s}-\kappa_{s}^{\prime} \alpha_{s}-\frac{1}{2} \kappa_{s}^{\prime} \Lambda_{s} \kappa_{s}\right) \mathrm{d} s-\int_{t}^{T}\left(\alpha_{s}+\Lambda_{s} \kappa_{s}\right) \mathrm{d} B_{s}^{\kappa} \\
& +\int_{t}^{T} \mathbb{1}_{\Upsilon_{1}}(s) v_{s} \mathrm{~d} B_{s}^{\kappa}-\frac{1}{2 \delta} \int_{t}^{T} \mathbb{1}_{\Upsilon_{1}}(s) \mathrm{d} s \\
\geq & G-\int_{t}^{T}\left(\chi_{s}-\kappa_{s}^{\prime} \alpha_{s}-\frac{1}{2} \kappa_{s}^{\prime} \Lambda_{s} \kappa_{s}\right) \mathrm{d} s-\int_{t}^{T}\left(\alpha_{s}+\Lambda_{s} \kappa_{s}\right) \mathrm{d} B_{s}^{\kappa} \\
& +\int_{t}^{T} \mathbb{1}_{\Upsilon_{1}}(s) v_{s} \mathrm{~d} B_{s}^{\kappa}-\int_{t}^{T} \frac{1}{2}\left(\mathbb{1}_{\Upsilon_{1}}(s) v_{s}\right)^{\prime} \Lambda_{s}^{-1}\left(\mathbb{1}_{\Upsilon_{1}}(s) v_{s}\right) \mathrm{d} s
\end{aligned}
$$

by the definition of $\Upsilon_{1}$. But we also have like in (3.9) that

$$
\begin{aligned}
\Gamma_{t}= & G-\int_{t}^{T}\left(\chi_{s}-\kappa_{s}^{\prime} \alpha_{s}-\frac{1}{2} \kappa_{s}^{\prime} \Lambda_{s} \kappa_{s}\right) \mathrm{d} s+\int_{t}^{T} Z_{s} \mathrm{~d} B_{s}^{\kappa} \\
& -\int_{t}^{T} \frac{1}{2}\left(Z_{s}+\alpha_{s}+\Lambda_{s} \kappa_{s}\right)^{\prime} \Lambda_{s}^{-1}\left(Z_{s}+\alpha_{s}+\Lambda_{s} \kappa_{s}\right) \mathrm{d} s,
\end{aligned}
$$

and subtracting (3.18) from (3.17), we obtain

$$
\begin{aligned}
& 0 \geq \int_{t}^{T}\left(\mathbb{1}_{\Upsilon_{1}}(s) v_{s}-\alpha_{s}-\Lambda_{s} \kappa_{s}-Z_{s}\right) \\
& \quad \times\left(\mathrm{d} B_{s}^{\kappa}-\Lambda_{s}^{-1}\left(\mathbb{1}_{\Upsilon_{1}}(s) v_{s}+\alpha_{s}+\Lambda_{s} \kappa_{s}+Z_{s}\right) \mathrm{d} s\right) .
\end{aligned}
$$

Like in the proof of Proposition 2.3, the right-hand side of (3.19) has zero expectation under some equivalent probability measure. Hence it must vanish, so we must also have equality in (3.17), and this implies $(P \otimes \operatorname{Leb})\left[\Upsilon_{1}\right]=0$. Analogously, we have 
$\left(P \otimes\right.$ Leb) $\left[\Upsilon_{2}\right]=0$ for

$$
\Upsilon_{2}:=\left\{(\omega, s) \in \rrbracket t, T \rrbracket \mid \max \operatorname{spec}\left(\Lambda_{s}(\omega)\right)>\delta\right\}
$$

This shows (3.14) with $c:=\delta$ and also gives the last assertion.

\subsection{Projecting the BSDE}

Let us split $B=(\bar{B}, \underline{B})^{\prime}$ into $\bar{B}$ and $\underline{B}$, an $\bar{n}$ - and an $\underline{n}$-dimensional $(\mathbb{F}, P)$-Brownian motion with $\bar{n}+\underline{n}=n$. What happens to the BSDE

$$
\Gamma_{s}=G-\int_{s}^{T}\left(f\left(\Lambda_{r}, Z_{r}+\alpha_{r}\right)+\chi_{r}\right) \mathrm{d} r+\int_{s}^{T} Z_{r} \mathrm{~d} B_{r}, \quad 0 \leq s \leq T
$$

if we project it, in a way to be specified, onto the filtration generated by $\bar{B}$ ? In this section, we precisely formulate and then answer this question.

Let $\overline{\mathbb{F}}=\left(\overline{\mathcal{F}}_{s}\right)_{0 \leq s \leq T}$ be the augmented filtration generated by $\bar{B}$. For a process $Z$, we denote its componentwise optional ( $P$-)projection onto $\overline{\mathbb{F}}$ by $Z^{o}$ (if it exists). It is-by definition-the unique $\overline{\mathbb{F}}$-optional process satisfying $Z_{\tau}^{o}=E\left[Z_{\tau} \mid \overline{\mathcal{F}}_{\tau}\right]$ for every $\overline{\mathbb{F}}$-stopping time $\tau$.

To compare (2.1) with a BSDE driven by $\bar{B}$, write $\alpha=(\bar{\alpha}, \underline{\alpha})^{\prime}$ and denote by $\bar{\Lambda}$ the upper-left $\bar{n} \times \bar{n}$ components of $\Lambda$. A solution, for $s \in[0, T]$, of

$$
\check{\Gamma}_{s}=E\left[G \mid \overline{\mathcal{F}}_{T}\right]-\int_{s}^{T}\left(\frac{1}{2}\left(\check{Z}_{r}+\bar{\alpha}_{r}^{o}\right)^{\prime}\left(\bar{\Lambda}_{r}^{o}\right)^{-1}\left(\check{Z}_{r}+\bar{\alpha}_{r}^{o}\right)+\chi_{r}^{o}\right) \mathrm{d} r+\int_{s}^{T} \check{Z}_{r} \mathrm{~d} \bar{B}_{r}
$$

is a pair $(\check{\Gamma}, \check{Z})$ satisfying (3.20), where $\check{\Gamma}$ is a real-valued bounded continuous $(\overline{\mathbb{F}}, P)$ semimartingale and $\check{Z}$ is an $\mathbb{R}^{\bar{n}}$-valued $\overline{\mathbb{F}}$-predictable process such that $\int_{0}^{T}\left|\check{Z}_{s}\right|^{2} \mathrm{~d} s<\infty$ almost surely. Note that $\bar{X}^{o}:=(\bar{X})^{o}=\overline{\left(X^{o}\right)}$ for $X=\alpha, \Lambda$.

Theorem 3.3 The BSDE (3.20) has a unique solution $(\check{\Gamma}, \check{Z})$. It satisfies $\Gamma^{o} \leq \check{\Gamma}$, where $(\Gamma, Z)$ is the solution of $(2.1)$.

Theorem 3.3 is a Jensen-type inequality for quadratic BSDEs. For a simple illustration, take $\bar{n}=\underline{n}=1$ and $\Lambda \equiv c I, \alpha \equiv 0, \chi \equiv 0$. In this case, the solution of (2.1) has $\Gamma_{0}=-c \log E[\exp (-G / c)]$ by (2.4), and analogously, we have $\check{\Gamma}_{0}=-c \log E\left[\exp \left(-\frac{1}{c} E\left[G \mid \overline{\mathcal{F}}_{T}\right]\right)\right]$. So $\Gamma_{0}^{o} \leq \check{\Gamma}_{0}$ follows here also directly from Jensen's inequality.

Proof of Theorem 3.3 As in Lemma 2.2, (3.20) has a unique solution $(\check{\Gamma}, \check{Z})$, and $\int \check{Z} \mathrm{~d} \bar{B} \in B M O(\overline{\mathbb{F}}, P)$. Fix $s \in[0, T]$ and condition (2.1) on $\overline{\mathcal{F}}_{s}$ to get 


$$
\begin{aligned}
E\left[\Gamma_{s} \mid \overline{\mathcal{F}}_{s}\right]=\Gamma_{0}+E\left[\int_{0}^{s}\left(f\left(\Lambda_{r}, Z_{r}+\alpha_{r}\right)+\chi_{r}\right) \mathrm{d} r \mid \overline{\mathcal{F}}_{s}\right] \\
-E\left[\int_{0}^{s} Z_{r} \mathrm{~d} B_{r} \mid \overline{\mathcal{F}}_{s}\right] .
\end{aligned}
$$

Note next that $\chi^{o}$ exists since $\chi$ is bounded by assumption. We claim that

$$
E\left[\int_{0}^{s} \chi_{r} \mathrm{~d} r \mid \overline{\mathcal{F}}_{s}\right]=\int_{0}^{s} \chi_{r}^{o} \mathrm{~d} r
$$

and because $\overline{\mathbb{F}}$ is generated by $\bar{B}$, it is by Itô's representation theorem enough to show that

$$
E\left[\int_{0}^{s} \chi_{r} \mathrm{~d} r \int_{0}^{s} \beta_{q} \mathrm{~d} \bar{B}_{q}\right]=E\left[\int_{0}^{s} \chi_{r}^{o} \mathrm{~d} r \int_{0}^{s} \beta_{q} \mathrm{~d} \bar{B}_{q}\right]
$$

for any $\overline{\mathbb{F}}$-predictable $\beta$ such that $\int \beta \mathrm{d} \bar{B}$ is bounded. By Fubini's theorem,

$$
E\left[\int_{0}^{s} \chi_{r} \mathrm{~d} r \int_{0}^{s} \beta_{q} \mathrm{~d} \bar{B}_{q}\right]=\int_{0}^{s} E\left[\chi_{r} \int_{0}^{s} \beta_{q} \mathrm{~d} \bar{B}_{q}\right] \mathrm{d} r
$$

and conditioning on $\overline{\mathcal{F}}_{r}$ for $r \in[0, s]$ yields

$$
E\left[\chi_{r} \int_{0}^{s} \beta_{q} \mathrm{~d} \bar{B}_{q}\right]=E\left[\chi_{r} \int_{0}^{r} \beta_{q} \mathrm{~d} \bar{B}_{q}\right]=E\left[\chi_{r}^{o} \int_{0}^{s} \beta_{q} \mathrm{~d} \bar{B}_{q}\right],
$$

which implies (3.23) by using (3.24) once for $\chi$ and once for $\chi^{o}$ instead of $\chi$. So we have (3.22), and using $f \geq 0$, we analogously obtain

$$
E\left[\int_{0}^{s} f\left(\Lambda_{r}, Z_{r}+\alpha_{r}\right) \mathrm{d} r \mid \overline{\mathcal{F}}_{s}\right]=\int_{0}^{s}(f(\Lambda, Z+\alpha))_{r}^{o} \mathrm{~d} r .
$$

To simplify the term $E\left[\int_{0}^{s} Z_{r} \mathrm{~d} B_{r} \mid \overline{\mathcal{F}}_{s}\right]$ in (3.21), we use the optional projection of $Z$. However, we cannot use the classical optional projection because $Z$ is in general neither bounded nor nonnegative. We define $Z^{o}$ instead by

$$
Z^{o}:=\left\{\begin{array}{cl}
\left(Z^{+}\right)^{o}-\left(Z^{-}\right)^{o} & \text { if }|Z|^{o}<\infty \\
0 & \text { otherwise }
\end{array}\right.
$$


where $Z^{ \pm}:=\left(\left(Z^{1}\right)^{ \pm}, \ldots,\left(Z^{n}\right)^{ \pm}\right)^{\prime}$. Then $Z^{o}$ is $\overline{\mathbb{F}}$-optional and $|Z|^{o}<\infty(P \otimes$ Leb)a.e. since Tonelli's theorem and $\int Z \mathrm{~d} B \in B M O(\mathbb{F}, P)$ by Lemma 2.2 give

$$
\int_{0}^{T} E\left[|Z|_{r}^{o}\right] \mathrm{d} r=\int_{0}^{T} E\left[\left|Z_{r}\right|\right] \mathrm{d} r=E\left[\int_{0}^{T}\left|Z_{r}\right| \mathrm{d} r\right]<\infty
$$

Write $Z=(\bar{Z}, \underline{Z})^{\prime}$ and $Z^{o}=\left(\bar{Z}^{o}, \underline{Z}^{o}\right)^{\prime}$. We then have

$$
E\left[\int_{0}^{s} Z_{r} \mathrm{~d} B_{r} \mid \overline{\mathcal{F}}_{s}\right]=\int_{s}^{T} \bar{Z}_{r}^{o} \mathrm{~d} \bar{B}_{r}
$$

indeed, using $E\left[\bar{Z}_{r} \mid \overline{\mathcal{F}}_{r}\right]=\bar{Z}_{r}^{o} P$-a.s. for Leb-a.a. $r \in[0, s]$ and the isometry property of the stochastic integral, we obtain similarly to (3.23) that

$$
E\left[\int_{0}^{s} Z_{r} \mathrm{~d} B_{r} \int_{0}^{s} \beta_{q} \mathrm{~d} \bar{B}_{q}\right]=E\left[\int_{0}^{s} \beta_{r}^{\prime} \bar{Z}_{r} \mathrm{~d} r\right]=E\left[\int_{0}^{s} \bar{Z}_{r}^{o} \mathrm{~d} \bar{B}_{r} \int_{0}^{s} \beta_{q} \mathrm{~d} \bar{B}_{q}\right]
$$

for any $\overline{\mathbb{F}}$-predictable $\beta$ such that $\int \beta \mathrm{d} \bar{B}$ is bounded, and this implies (3.26) by Itô's representation theorem. Combining (3.21), (3.22), (3.25) and (3.26) thus yields

$$
E\left[\Gamma_{s} \mid \overline{\mathcal{F}}_{S}\right]=E\left[G \mid \overline{\mathcal{F}}_{T}\right]-\int_{S}^{T}\left((f(\Lambda, Z+\alpha))_{r}^{o}+\chi_{r}^{o}\right) \mathrm{d} r+\int_{s}^{T} \bar{Z}_{r}^{o} \mathrm{~d} \bar{B}_{r} .
$$

Due to Lemma A.1, the function $f$ is jointly convex. Identifying $(A, z)$ in $\mathcal{S}^{n} \times \mathbb{R}^{n}$ with a vector in $\mathbb{R}^{\frac{n(n+1)}{2}+n}$, we view $f$ as a function on such vectors and apply Jensen's inequality to obtain for any $\overline{\mathbb{F}}$-stopping time $\tau$ that

$$
(f(\Lambda, Z+\alpha))_{\tau}^{o}=E\left[f\left(\Lambda_{\tau}, Z_{\tau}+\alpha_{\tau}\right) \mid \overline{\mathcal{F}}_{\tau}\right] \geq f\left(\Lambda_{\tau}^{o}, Z_{\tau}^{o}+\alpha_{\tau}^{o}\right) \mathbb{1}_{|Z|_{\tau}^{o}<\infty}
$$

Thus the optional section theorem and $|Z|^{o}<\infty(P \otimes$ Leb)-a.e. yield

$$
(f(\Lambda, Z+\alpha))^{o} \geq f\left(\Lambda^{o}, Z^{o}+\alpha^{o}\right) \mathbb{1}_{|Z|^{o}<\infty}=f\left(\Lambda^{o}, Z^{o}+\alpha^{o}\right)
$$

( $P \otimes$ Leb)-a.e. A simple calculation (see Remark 3.4 below) shows that

$$
f(A, z)=\frac{1}{2} z^{\prime} A^{-1} z \geq \frac{1}{2} \bar{z}^{\prime}(\bar{A})^{-1} \bar{z}
$$


for any $A \in \mathcal{S}^{n}$ and $z=(\bar{z}, \underline{z})^{\prime} \in \mathbb{R}^{n}$, with $\bar{A}$ denoting the upper-left $\bar{n} \times \bar{n}$ components of $A$. In view of (3.27), we obtain from (3.28) and (3.29) that

$$
\begin{aligned}
E\left[\Gamma_{s} \mid \overline{\mathcal{F}}_{s}\right] \leq & E\left[G \mid \overline{\mathcal{F}}_{T}\right]-\int_{s}^{T}\left(\frac{1}{2}\left(\bar{Z}_{r}^{o}+\bar{\alpha}_{r}^{o}\right)^{\prime}\left(\bar{\Lambda}_{r}^{o}\right)^{-1}\left(\bar{Z}_{r}^{o}+\bar{\alpha}_{r}^{o}\right)+\chi_{r}^{o}\right) \mathrm{d} r \\
& +\int_{s}^{T} \bar{Z}_{r}^{o} \mathrm{~d} \bar{B}_{r} .
\end{aligned}
$$

Hence (3.20) implies

$$
\begin{aligned}
E\left[\Gamma_{s} \mid \overline{\mathcal{F}}_{s}\right]-\check{\Gamma}_{s} \leq & -\int_{s}^{T} \frac{1}{2}\left(\bar{Z}_{r}^{o}-\check{Z}_{r}\right)^{\prime}\left(\bar{\Lambda}_{r}^{o}\right)^{-1}\left(\bar{Z}_{r}^{o}+\check{Z}_{r}+2 \bar{\alpha}_{r}^{o}\right) \mathrm{d} r \\
& +\int_{s}^{T}\left(\bar{Z}_{r}^{o}-\check{Z}_{r}\right) \mathrm{d} \bar{B}_{r} .
\end{aligned}
$$

We know that $\int \check{Z} \mathrm{~d} \bar{B}$ is in $B M O(\overline{\mathbb{F}}, P)$, and so is $\int \bar{Z}^{o} \mathrm{~d} \bar{B}$ because $\int Z \mathrm{~d} B$ is in $B M O(\mathbb{F}, P)$. Like in the proof of Proposition 2.3, we deduce that $E\left[\Gamma_{s} \mid \overline{\mathcal{F}}_{s}\right] \leq \check{\Gamma}_{s}$ for $s \in[0, T]$, and this concludes the proof because $\check{\Gamma}, \Gamma$ and hence $\Gamma^{o}$ are continuous.

Remark 3.4 (1) As the proof shows, we do not need for Theorem 3.3 that the generator of the BSDE (2.1) is purely quadratic like $f$ in (2.2). We only need that it is jointly convex, satisfies a quadratic growth condition and dominates the generator of the projected BSDE (3.20). In particular, Theorem 3.3 also applies for a generator $\tilde{f}$ of the form $\tilde{f}(A, z)=\frac{1}{2} \bar{z}^{\prime} \bar{A}^{-1} \bar{z}$ with $\bar{z}$ and $\bar{A}$ as in (3.29). This will later be used in the applications to indifference valuation.

(2) In linear algebra, the shorted operator $\operatorname{sh}: \mathcal{S}^{n} \rightarrow \mathcal{S}^{\bar{n}}$ is defined by

$$
\operatorname{sh}(A):=A^{11}-A^{12}\left(A^{22}\right)^{-1}\left(A^{12}\right)^{\prime} \text { for } A=\left(\begin{array}{cc}
A^{11} & A^{12} \\
\left(A^{12}\right)^{\prime} & A^{22}
\end{array}\right) \in \mathcal{S}^{n}
$$

One can check that $\left(A^{11}\right)^{-1}=\operatorname{sh}\left(A^{-1}\right)$ and verify by completion of squares that

$$
\bar{z}^{\prime} \operatorname{sh}(A) \bar{z}=\min _{\underline{z} \in \mathbb{R}^{n}}\left(\left(\bar{z}^{\prime}, \underline{z}^{\prime}\right) A\left(\begin{array}{l}
\bar{z} \\
\underline{z}
\end{array}\right)\right) \quad \text { for } \bar{z} \in \mathbb{R}^{\bar{n}} \text { and } A \in \mathcal{S}^{n}
$$

The inequality (3.29) follows immediately. 


\subsection{Symmetrising the BSDE}

This section establishes our third main result, Theorem 3.7, giving an explicit upper bound for the solution $\Gamma$ of (2.1). We first study how the BSDE (2.1) is affected by orthogonal transformations on the underlying probability space. To have some structure, we work on Wiener space, i.e., take $\Omega:=C\left([0, T], \mathbb{R}^{n}\right)$ with the Borel $\sigma$-field $\mathcal{F}$ and Wiener measure $P$ so that the coordinate process $B$ is a $P$-Brownian motion. Recall that $t \in[0, T]$ is fixed.

For an orthogonal $(n \times n)$-matrix, $u \in \mathrm{O}(n)$, we define a corresponding mapping $U_{t}: C\left([0, T], \mathbb{R}^{n}\right) \rightarrow C\left([0, T], \mathbb{R}^{n}\right)$ by applying $u$ from time $t$ on, i.e.,

$$
U_{t}(g)(s)=\left\{\begin{array}{ll}
g(s) & \text { if } s \leq t, \\
g(t)+u(g(s)-g(t)) & \text { if } s>t,
\end{array} \text { for } g \in C\left([0, T], \mathbb{R}^{n}\right) .\right.
$$

Then $B^{u}:=U_{t} \circ B$ is an $\mathbb{R}^{n}$-valued $(\mathbb{F}, P)$-Brownian motion since $u$ is orthogonal. The following result says that if one transforms by $U_{t}$ the driver and the terminal value of a BSDE, the solution of the new BSDE is the $U_{t}$-transformation of the original solution. This is very intuitive and analogous to orthogonally transforming the variables in a second-order PDE; compare Sect. 2.2. The reason why this also works for BSDEs is that $B \circ U_{t}=U_{t} \circ B=B^{u}$, i.e., Brownian motion and the transformation $U_{t}$ commute on Wiener space.

Lemma 3.5 Let $u \in \mathrm{O}(n)$ and assume that the $B S D E$

$$
\Gamma_{s}=G-\int_{s}^{T} F_{r}\left(\Gamma_{r}, Z_{r}\right) \mathrm{d} r+\int_{s}^{T} Z_{r} \mathrm{~d} B_{r}, \quad 0 \leq s \leq T
$$

for a general $\mathbb{F}$-predictable $F: \mathbb{R}^{n+1} \rightarrow \mathbb{R}$ has a unique solution $(\Gamma, Z)$ (in the sense of Sect. 2.1). Then $\left(\Gamma \circ U_{t}, Z \circ U_{t}\right)$ is the unique solution of

$$
\tilde{\Gamma}_{s}=G \circ U_{t}-\int_{s}^{T}\left(F \circ U_{t}\right)_{r}\left(\tilde{\Gamma}_{r}, \tilde{Z}_{r}\right) \mathrm{d} r+\int_{s}^{T} \tilde{Z}_{r} \mathrm{~d} B_{r}^{u}, \quad 0 \leq s \leq T .
$$

In particular, the solution $\left(\Gamma \circ U_{t}, Z \circ U_{t}\right)$ of (3.31) coincides on $\llbracket 0, t \rrbracket$ with the solution $(\Gamma, Z)$ of $(3.30)$.

Proof Let $(\Gamma, Z)$ be the solution of (3.30) and define $\tilde{\Gamma}$ for $0 \leq s \leq T$ by

$$
\begin{aligned}
\tilde{\Gamma}_{s} & :=\Gamma_{0}+\int_{0}^{s}\left(F \circ U_{t}\right)_{r}\left(\Gamma_{r} \circ U_{t}, Z_{r} \circ U_{t}\right) \mathrm{d} r-\int_{0}^{s}\left(Z_{r} \circ U_{t}\right) \mathrm{d} B_{r}^{u} \\
& =\Gamma_{0}+\left(\int_{0}^{s} F_{r}\left(\Gamma_{r}, Z_{r}\right) \mathrm{d} r\right) \circ U_{t}-\int_{0}^{s}\left(Z_{r} \circ U_{t}\right) \mathrm{d} B_{r}^{u} .
\end{aligned}
$$


In Lemma A.3 in the Appendix, we prove that, as one expects,

$$
\int\left(Z \circ U_{t}\right) \mathrm{d} B^{u}=\left(\int Z \mathrm{~d} B\right) \circ U_{t} .
$$

This gives by (3.30) that $\tilde{\Gamma}=\Gamma \circ U_{t}$ and thus ( $\left.\Gamma \circ U_{t}, Z \circ U_{t}\right)$ solves (3.31). Uniqueness for (3.31) follows since $U_{t}$ is bijective; indeed, if $(\tilde{\Gamma}, \tilde{Z})$ solves $(3.31)$, then (3.32) and (3.33) imply that $\left(\tilde{\Gamma} \circ U_{t}^{-1}, \tilde{Z} \circ U_{t}^{-1}\right)$ solves (3.30) whose unique solution is $(\Gamma, Z)$.

The next proposition states that averaging in $\omega$ over a set of orthogonal transformations increases the solution of (2.1).

Proposition 3.6 Take a finite subset $\mathcal{O}$ of $\mathrm{O}(n)$ with cardinality $|\mathcal{O}|$ and set

$$
\begin{array}{rlrl}
G^{\mathcal{O}} & :=\frac{1}{|\mathcal{O}|} \sum_{u \in \mathcal{O}} G \circ U_{t}, & \Lambda^{\mathcal{O}}:=\frac{1}{|\mathcal{O}|} \sum_{u \in \mathcal{O}} u^{\prime}\left(\Lambda \circ U_{t}\right) u, \\
\alpha^{\mathcal{O}}:=\frac{1}{|\mathcal{O}|} \sum_{u \in \mathcal{O}} u^{\prime}\left(\alpha \circ U_{t}\right), \quad \chi^{\mathcal{O}}:=\frac{1}{|\mathcal{O}|} \sum_{u \in \mathcal{O}} \chi \circ U_{t} .
\end{array}
$$

Then the solutions $(\Gamma, Z)$ of (2.1) and $\left(\Gamma^{\mathcal{O}}, Z^{\mathcal{O}}\right)$, for $0 \leq s \leq T$, of

$$
\tilde{\Gamma}_{s}=G^{\mathcal{O}}-\int_{s}^{T}\left(f\left(\Lambda_{r}^{\mathcal{O}}, \tilde{Z}_{r}+\alpha_{r}^{\mathcal{O}}\right)+\chi_{r}^{\mathcal{O}}\right) \mathrm{d} r+\int_{s}^{T} \tilde{Z}_{r} \mathrm{~d} B_{r}
$$

satisfy $\Gamma_{t} \leq \Gamma_{t}^{\mathcal{O}}$ almost surely.

Proof By Lemma 2.2, (2.1) and (3.34) have unique solutions. For $u \in \mathcal{O}$, we denote by $\left(\Gamma^{u}, Z^{u}\right)$ the solution of (2.1) corresponding to the parameters $\left(G \circ U_{t}, u^{\prime}\left(\Lambda \circ U_{t}\right) u, u^{\prime}\left(\alpha \circ U_{t}\right), \chi \circ U_{t}\right)$. The concavity from Proposition 2.3 gives $\Gamma^{\mathcal{O}}=\Gamma\left(G^{\mathcal{O}}, \Lambda^{\mathcal{O}}, \alpha^{\mathcal{O}}, \chi^{\mathcal{O}}\right) \geq \frac{1}{|\mathcal{O}|} \sum_{u \in \mathcal{O}} \Gamma^{u}$, and so it is enough to show $\Gamma_{t}^{u}=\Gamma_{t}$ for every $u \in \mathcal{O}$. Fix $u \in \mathcal{O}$. Applying Lemma 3.5 to (2.1) yields that the solution of the $U_{t}$-transformed BSDE is $(\tilde{\Gamma}, \tilde{Z}):=\left(\Gamma \circ U_{t}, Z \circ U_{t}\right)$. Setting $\hat{Z}:=u^{\prime} \tilde{Z}$ and using $\hat{Z} \mathrm{~d} B=\tilde{Z} \mathrm{~d} B^{u}$ and, due to (2.2),

$$
f\left(\Lambda \circ U_{t}, \tilde{Z}+\alpha \circ U_{t}\right)=f\left(u^{\prime}\left(\Lambda \circ U_{t}\right) u, \hat{Z}+u^{\prime}\left(\alpha \circ U_{t}\right)\right)
$$

we obtain that the $U_{t}$-transformed BSDE is, for $t \leq s \leq T$, equivalent to

$$
\begin{aligned}
\tilde{\Gamma}_{s}= & G \circ U_{t}-\int_{s}^{T}\left(f\left(u^{\prime}\left(\Lambda \circ U_{t}\right)_{r} u, \hat{Z}_{r}+u^{\prime}\left(\alpha \circ U_{t}\right)_{r}\right)+\left(\chi \circ U_{t}\right)_{r}\right) \mathrm{d} r \\
& +\int_{s}^{T} \hat{Z}_{r} \mathrm{~d} B_{r} .
\end{aligned}
$$


But this is (2.1) with the parameters $\left(G \circ U_{t}, u^{\prime}\left(\Lambda \circ U_{t}\right) u, u^{\prime}\left(\alpha \circ U_{t}\right), \chi \circ U_{t}\right)$. So $\Gamma^{u}=\tilde{\Gamma}=\Gamma \circ U_{t}$ on $\llbracket t, T \rrbracket$ and thus $\Gamma_{t}^{u}=\Gamma_{t}$, since $\Gamma \circ U_{t}=\Gamma$ on $\llbracket 0, t \rrbracket$.

The idea to exploit Proposition 3.6 is now that choosing a "good" set $\mathcal{O}$ yields with (3.34) an easier BSDE than the original one in (2.1), so that an upper bound for the solution $(\Gamma, Z)$ of (2.1) becomes more explicit. By Theorem 3.1, the upper bound for $\Gamma$ is increasing in the maximal eigenvalue, $\max \operatorname{spec}(\Lambda)$. Assume for the moment that $\Lambda$ is deterministic. If we first apply Proposition 3.6 to (2.1) and then Theorem 3.1 to (3.34), we obtain an upper bound depending on $\max \operatorname{spec}\left(\frac{1}{|\mathcal{O}|} \sum_{u \in \mathcal{O}} u^{\prime} \Lambda u\right)$. A simple calculation shows that for any matrix $A \in \mathcal{S}^{n}$ and finite subset $\mathcal{O}$ of $\mathrm{O}(n)$,

$$
\frac{1}{n} \operatorname{tr}(A) \leq \max \operatorname{spec}\left(\frac{1}{|\mathcal{O}|} \sum_{u \in \mathcal{O}} u^{\prime} A u\right) \leq \max \operatorname{spec}(A),
$$

and so we obtain a smaller distortion power $\delta_{t}^{\max }$ by averaging over $\mathcal{O}$. On the other hand, however, averaging $G, \alpha$ and $\chi$ may worsen the bound on $\Gamma$, and an example in Sect. 5.3 shows how these two effects interact. The best lower bound for $\max \operatorname{spec}(A)$ that we can obtain by averaging over $\mathcal{O}$ is $\frac{1}{n} \operatorname{tr}(A)$ by (3.35), and if $A$ is diagonal, this is attained for $\mathcal{O}=$ Perm, the symmetric group of permutations of length $n$. (We identify permutations with corresponding orthogonal matrices and use

$$
\left.\frac{1}{\mid \text { Perm } \mid} \sum_{u \in \text { Perm }} u^{\prime} A u=\frac{\operatorname{tr}(A)}{n} I \text { for any diagonal matrix } A .\right)
$$

The idea to choose $\mathcal{O}=$ Perm leads us to the next result.

Theorem 3.7 Assume that $\Lambda=\left(\Lambda^{i j}\right)_{i, j=1, \ldots, n}$ is a diagonal matrix, and define

$$
\begin{array}{rlrl}
G^{\text {Sym }} & :=\frac{1}{n !} \sum_{u \in \text { Perm }} G \circ U_{t}, & d_{t} & :=\sup _{s \in[t, T]}\left\|\frac{1}{n} \sum_{j=1}^{n} \max _{u \in \operatorname{Perm}}\left(\Lambda_{s}^{j j} \circ U_{t}\right)\right\|_{L^{\infty}}, \\
\alpha^{\text {Sym }} & :=\frac{1}{n !} \sum_{u \in \text { Perm }} u^{\prime}\left(\alpha \circ U_{t}\right), \quad \chi^{\text {Sym }}:=\frac{1}{n !} \sum_{u \in \text { Perm }} \chi \circ U_{t} .
\end{array}
$$

Then the solution $(\Gamma, Z)$ of (2.1) satisfies

$$
\Gamma_{t} \leq-d_{t} \log E\left[\exp \left(-G^{\operatorname{Sym}}+\int_{t}^{T} \alpha_{s}^{\operatorname{Sym}} \mathrm{d} B_{s}+\int_{t}^{T} \chi_{s}^{\operatorname{Sym}} \mathrm{d} s\right)^{\frac{1}{d_{t}}} \mid \mathcal{F}_{t}\right]
$$

Proof By choosing $\mathcal{O}:=$ Perm, we obtain from Proposition 3.6 a first upper bound $\Gamma_{t} \leq \Gamma_{t}^{\mathcal{O}}$, where $\Gamma_{t}^{\mathcal{O}}$ solves the BSDE (3.34) with $\mathcal{O}:=$ Perm. We now apply 
Theorem 3.1 to $\Gamma^{\mathcal{O}}$ with $\kappa \equiv 0$, which gives

$$
\Gamma_{t} \leq-\bar{\delta}_{t} \log E\left[\exp \left(-G^{\mathrm{Sym}}+\int_{t}^{T} \alpha_{s}^{\mathrm{Sym}} \mathrm{d} B_{s}+\int_{t}^{T} \chi_{s}^{\mathrm{Sym}} \mathrm{d} s\right)^{\frac{1}{\delta_{t}}} \mid \mathcal{F}_{t}\right]
$$

with

$$
\bar{\delta}_{t}:=\sup _{s \in[t, T]}\left\|\max \operatorname{spec}\left(\frac{1}{n !} \sum_{u \in \operatorname{Perm}} u^{\prime}\left(\Lambda_{s} \circ U_{t}\right) u\right)\right\|_{L^{\infty}} \leq d_{t}
$$

since $\Lambda$ is diagonal. Thus (3.36) follows from Jensen's inequality.

The assumption that $\Lambda$ is diagonal is less restrictive than it looks. We can always rewrite (2.1) to another BSDE of the same type with diagonal $\Lambda$ by changing $\alpha$ and $B$. In fact, there exist a predictable $\mathrm{O}(n)$-valued process $O$ and a predictable diagonal matrix $D$ such that $\Lambda=O^{\prime} D O$. If we now define an $(\mathbb{F}, P)$-Brownian motion by $\mathrm{d} B^{O}=O \mathrm{~d} B$, a direct calculation shows that if $(\Gamma, Z)$ solves $(2.1)$ with parameters $(G, \Lambda, \alpha, \chi)$, then $(\Gamma, O Z)$ solves $(2.1)$ with parameters $(G, D, O \alpha, \chi)$ and with $B$ replaced by $B^{O}$. This reduces the problem to the case of a diagonal matrix $\Lambda$, but we then have to symmetrise with respect to $B^{O}$ and not $B$. For this, $G, \alpha$ and $\chi$ must be measurable for the filtration $\mathbb{F}^{O}$ generated by $B^{O}$, which can be smaller than $\mathbb{F}$. This limitation does not come up if $\Lambda$ is deterministic, since then so is $O$ and hence $\mathbb{F}^{O}=\mathbb{F}$. In Sect. 5, we relate the BSDE (2.1) to an optimisation problem where the matrix $\Lambda$ is a transform of the correlation matrix of certain price processes. In applications, such matrices are often assumed to be deterministic. Similarly, things typically become less restrictive in a Markovian setting because one can often do everything in the filtration of the factor process.

Remark 3.8 One can generalise Theorems 3.3 and 3.7 to the case where $G, \alpha$ and $\chi$ are unbounded, but $|G|$ and $\int_{0}^{T}\left(\left|\alpha_{s}\right|^{2}+\left|\chi_{s}\right|\right) \mathrm{d} s$ have exponential moments of all order. We sketch the procedure for such a generalisation. One first uses Corollary 6 of Briand and $\mathrm{Hu}$ [5] for the existence of a generalised solution $(\Gamma, Z)$ of (2.1) and its uniqueness in a suitable class. Then one sets $G^{j}:=G^{+} \wedge j-G^{-} \wedge j, j \in \mathbb{N}$, defines $\alpha^{j}$ and $\chi^{j}$ analogously, and applies Theorems 3.3 and 3.7 when $G, \alpha$ and $\chi$ are replaced by $G^{j}, \alpha^{j}$ and $\chi^{j}$. By taking limits in a suitable sense and applying Proposition 7 of Briand and $\mathrm{Hu}$ [5], one can deduce generalised versions of Theorems 3.3 and 3.7. We do not know whether Theorem 3.1 can also be formulated for unbounded $G, \alpha$ and $\chi$, because the above generalisation procedure does not work there.

One cannot weaken in the above way the assumption that the eigenvalues of $\Lambda$ are bounded away from zero, since this condition is needed to apply the results of Briand and $\mathrm{Hu}$ [5]. However, one can get rid of the restriction that the eigenvalues of $\Lambda$ are bounded away from infinity. Theorems 3.1 and 3.7 can be formulated without this assumption similarly to Theorem 4 of Frei and Schweizer [8]. If the componentwise optional projection of $\Lambda$, whose eigenvalues are not bounded away from infinity, exists $(P \otimes$ Leb)-a.e., one can prove Theorem 3.3 in the same way as in Sect. 3.2. 


\section{Exponential utility indifference valuation}

This section recalls the financial concept of indifference valuation, in preparation for applying the convexity results from Sect. 3 .

We work on a finite time interval $[0, T]$ for a fixed $T>0$, and we fix $t \in[0, T]$. On a complete probability space $(\Omega, \mathcal{G}, P)$, we have independent Brownian motions $W$ and $W^{\perp}$ with values in $\mathbb{R}^{m}$ and $\mathbb{R}^{n}$. We denote by $\mathbb{G}=\left(\mathcal{G}_{S}\right)_{0 \leq s \leq T}$ the $P$-augmented filtration generated by $\left(W, W^{\perp}\right)$ and assume $\mathcal{G}=\mathcal{G}_{T}$. Moreover, we suppose there is an $\mathbb{R}^{n}$-valued $(\mathbb{G}, P$ )-Brownian motion $Y$ such that

$$
\mathrm{d} Y_{s}=R_{s} \mathrm{~d} W_{s}+\sqrt{I-R_{S} R_{s}^{\prime}} \mathrm{d} W_{s}^{\perp}, \quad 0 \leq s \leq T
$$

for a $\mathbb{G}$-predictable $(n \times m)$-matrix $R$ describing correlations between $W$ and $Y$. We assume that all eigenvalues of $R R^{\prime}$ are bounded away from one uniformly on $\Omega \times[0, T]$, i.e., there exists $c \in[0,1)$ with

$$
\max \operatorname{spec}\left(R R^{\prime}\right) \leq c(P \otimes \text { Leb }) \text {-a.e. on } \Omega \times[0, T]
$$

For a fixed $\gamma>0$, the $\mathcal{S}^{n}$-valued process

$$
\Lambda=\frac{1}{\gamma}\left(I-R R^{\prime}\right)^{-1}
$$

is well defined, $\mathbb{G}$-predictable and satisfies $\operatorname{spec}(\Lambda) \subseteq\left[\frac{1}{\gamma}, \frac{1}{\gamma(1-c)}\right]$. In the notation of Sect. 3, this implies that $\delta_{t}^{\min }(\Lambda) \geq 1 / \gamma$.

Our financial market consists of a risk-free bank account yielding zero interest and $m$ traded risky assets $S=\left(S^{j}\right)_{j=1, \ldots, m}$ with dynamics

$$
\mathrm{d} S_{s}^{j}=S_{s}^{j} \mu_{s}^{j} \mathrm{~d} s+\sum_{k=1}^{m} S_{s}^{j} \sigma_{s}^{j k} \mathrm{~d} W_{s}^{k}, \quad 0 \leq s \leq T, S_{0}^{j}>0, \quad j=1, \ldots, m
$$

the drift vector $\mu=\left(\mu^{j}\right)_{j=1, \ldots, m}$ and the volatility matrix $\sigma=\left(\sigma^{j k}\right)_{j, k=1, \ldots, m}$ are $\mathbb{G}$-predictable. We assume that $\sigma$ is invertible, $\lambda:=\sigma^{-1} \mu$ is bounded (uniformly in $s$ and $\omega$ ) and that there exists a constant $C$ such that

$$
C \beta^{\prime} \beta \geq \beta^{\prime} \sigma \sigma^{\prime} \beta \geq \frac{1}{C} \beta^{\prime} \beta \text { on } \Omega \times[0, T] \text { for all } \beta \in \mathbb{R}^{m}
$$

(In other words, $\sigma$ is uniformly both bounded and elliptic.) The processes

$$
\hat{W}:=W+\int \lambda \mathrm{d} s \text { and } \hat{Y}:=Y+\int R \lambda \mathrm{d} s
$$


are Brownian motions under the minimal martingale measure $\hat{P}$ given by

$$
\frac{\mathrm{d} \hat{P}}{\mathrm{~d} P}:=\mathcal{E}\left(-\int \lambda \mathrm{d} W\right)_{T}
$$

Let $G$ be a bounded $\mathcal{G}_{T}$-measurable random variable, interpreted as a contingent claim or payoff due at time $T$. To value $G$, we assume that our investor has an exponential utility function $U(x)=-\exp (-\gamma x), x \in \mathbb{R}$, for a fixed $\gamma>0$. He starts at time $t$ with bounded $\mathcal{G}_{t}$-measurable initial capital $x_{t}$ and runs a self-financing strategy $\pi=\left(\pi_{s}\right)_{t \leq s \leq T}$ so that his wealth at time $s \in[t, T]$ is

$$
X_{s}^{x_{t}, \pi}=x_{t}+\int_{t}^{s} \sum_{j=1}^{m} \frac{\pi_{r}^{j}}{S_{r}^{j}} \mathrm{~d} S_{r}^{j}=x_{t}+\int_{t}^{s} \pi_{r}^{\prime} \sigma_{r} \mathrm{~d} \hat{W}_{r},
$$

where $\pi^{j}$ represents the amount invested in $S^{j}, j=1, \ldots, m$. The set $\mathcal{A}_{t}$ of admissible strategies on $[t, T]$ consists of all $\mathbb{G}$-predictable $\mathbb{R}^{m}$-valued processes $\pi=\left(\pi_{s}\right)_{t \leq s \leq T}$ which satisfy $\int_{t}^{T}\left|\pi_{s}\right|^{2} \mathrm{~d} s<\infty$ a.s. and are such that

$$
\exp \left(-\gamma X_{s}^{x_{t}, \pi}\right), t \leq s \leq T, \text { is of class }(D) \text { on }\left(\Omega, \mathcal{G}_{T}, \mathbb{G}, P\right)
$$

We define $V^{G}$ (and analogously $V^{0}$ ) by

$$
\begin{aligned}
V_{t}^{G}\left(x_{t}\right) & :=\underset{\pi \in \mathcal{A}_{t}}{\operatorname{ess} \sup _{P}} E_{P}\left[U\left(X_{T}^{x_{t}, \pi}+G\right) \mid \mathcal{G}_{t}\right] \\
& =\mathrm{e}^{-\gamma x_{t}} \underset{\pi \in \mathcal{A}_{t}}{\operatorname{ess} \sup _{P} E_{P}}\left[-\exp \left(-\gamma \int_{t}^{T} \pi_{s}^{\prime} \sigma_{s} \mathrm{~d} \hat{W}_{s}-\gamma G\right) \mid \mathcal{G}_{t}\right]
\end{aligned}
$$

so that $V_{t}^{G}\left(x_{t}\right)$ is the maximal expected utility the investor can achieve by starting at time $t$ with initial capital $x_{t}$, using some admissible strategy $\pi$, and receiving $G$ at time $T$. For ease of notation, we write

$$
V_{t}^{G}\left(x_{t}\right)=\mathrm{e}^{-\gamma x_{t}} V_{t}^{G}(0)=: \mathrm{e}^{-\gamma x_{t}} V_{t}^{G}
$$

Viewed over time, $V^{G}=\left(V_{t}^{G}\right)_{0 \leq t \leq T}$ is then the dynamic value process for the stochastic control problem associated to exponential utility maximisation.

The time $t$ indifference (buyer) value $b_{t}\left(x_{t}\right)$ for $G$ is implicitly defined by

$$
V_{t}^{0}\left(x_{t}\right)=V_{t}^{G}\left(x_{t}-b_{t}\left(x_{t}\right)\right) \text {. }
$$

This says that the investor is indifferent between solely trading with initial capital $x_{t}$, versus trading with reduced initial capital $x_{t}-b_{t}\left(x_{t}\right)$ but receiving $G$ at $T$. Our goal is to find bounds for $b_{t}\left(x_{t}\right)$. By (4.6), 


$$
b_{t}\left(x_{t}\right)=b_{t}=\frac{1}{\gamma} \log \frac{V_{t}^{0}}{V_{t}^{G}}
$$

does not depend on $x_{t}$, but directly on $V_{t}^{G}$ and $V_{t}^{0}$. We consider here $V_{t}^{0}$ as fixed via the financial market, and our focus lies on finding $G$-dependent bounds for $V^{G}$ from the optimisation problem (4.6). An overview of the literature on exponential utility indifference valuation in Brownian settings can be found in Section 4.2 of Frei and Schweizer [7].

\section{Valuation bounds from convexity}

In this section, we consider the same setup as in Sect. 4. In order to apply the convexity results from Sect. 3, we want to associate $V^{G}$ to a quadratic convex BSDE of the form (2.1). We start with the following result which follows directly from Theorem 7 and Proposition 9 of $\mathrm{Hu}$ et al. [9].

\section{Lemma 5.1 The BSDE}

$$
\check{\Gamma}_{s}=G-\int_{s}^{T}\left(\frac{\gamma}{2}\left|\check{Z}_{r}\right|^{2}-\hat{Z}_{r}^{\prime} \lambda_{r}-\frac{1}{2 \gamma}\left|\lambda_{r}\right|^{2}\right) \mathrm{d} r+\int_{s}^{T} \hat{Z}_{r} \mathrm{~d} W_{r}+\int_{s}^{T} \check{Z}_{r} \mathrm{~d} W_{r}^{\perp}
$$

for $s \in[0, T]$ has a unique solution $(\check{\Gamma}, \hat{Z}, \check{Z})$ such that $(\hat{Z}, \check{Z})$ is $\left(\mathbb{R}^{m} \times \mathbb{R}^{n}\right)$-valued and $\mathbb{G}$-predictable with $E_{P}\left[\int_{0}^{T}\left(\left|\hat{Z}_{s}\right|^{2}+\left|\check{Z}_{S}\right|^{2}\right) \mathrm{d} s\right]<\infty$ and $\check{\Gamma}$ is $\mathbb{G}$-predictable and bounded. Furthermore, we have $V_{\tau}^{G}=-\exp \left(-\gamma \check{\Gamma}_{\tau}\right)$ for any $\mathbb{G}$-stopping time $\tau$.

Unfortunately, we cannot (yet) apply the results from Sect. 3 to the BSDE (5.1), because its generator is quadratic in $\hat{Z}$, but only linear in $\hat{Z}$. In contrast, the generator of (2.1) is quadratic in the full vector $Z=(\hat{Z}, \hat{Z})^{\prime}$. The next sections present three different approaches to circumvent this problem. In Sect. 5.1, we simply add a term $\epsilon|\hat{Z}|^{2}$ to the generator of (5.1) and study the limit as $\epsilon$ tends to zero. Section 5.2 exploits the fact, pointed out in Remark 3.4, that one can apply the projection result in Theorem 3.3 to a BSDE with a more general generator. In a third approach, we impose in Sect. 5.3 measurability assumptions on the claim $G$ and the coefficients of the asset $S$ and then use symmetrisation arguments.

Lemma 5.1 also shows that the dynamic value process $V^{G}$ has a continuous version. In the sequel, we always use this version of $V^{G}$.

\section{$5.1 \epsilon$-Regularising the BSDE and changing the measure}

In this approach, we add a term $\epsilon|\hat{Z}|^{2}$ to the generator of (5.1) to bring it to the form of (2.1). In some sense, this makes the BSDE (5.1) more regular. We first study how the solution of the changed BSDE behaves as $\epsilon \searrow 0$. 
Lemma 5.2 For each fixed $\epsilon>0$, the BSDE

$$
\begin{aligned}
\check{\Gamma}_{s}^{\epsilon}= & G-\int_{s}^{T}\left(\frac{\gamma}{2}\left|\check{Z}_{r}^{\epsilon}\right|^{2}+\epsilon\left|\hat{Z}_{r}^{\epsilon}\right|^{2}-\left(\hat{Z}_{r}^{\epsilon}\right)^{\prime} \lambda_{r}-\frac{1}{2 \gamma}\left|\lambda_{r}\right|^{2}\right) \mathrm{d} r \\
& +\int_{s}^{T} \hat{Z}_{r}^{\epsilon} \mathrm{d} W_{r}+\int_{s}^{T} \check{Z}_{r}^{\epsilon} \mathrm{d} W_{r}^{\perp}, \quad 0 \leq s \leq T,
\end{aligned}
$$

has a unique solution $\left(\check{\Gamma}^{\epsilon}, \hat{Z}^{\epsilon}, \check{Z}^{\epsilon}\right)$ (in the sense of Lemma 5.1 ). The solution $\check{\Gamma}$ to (5.1) satisfies

$$
\check{\Gamma}_{t}=\underset{\epsilon>0}{\operatorname{ess} \sup } \check{\Gamma}_{t}^{\epsilon}=\lim _{\epsilon \searrow 0} \check{\Gamma}_{t}^{\epsilon} \text { a.s. }
$$

Lemma 5.2 is a variation of Proposition 3.1 of El Karoui et al. [6], which gives a similar conclusion for BSDEs with a Lipschitz-continuous generator.

Proof Lemma 2.2 gives for each $\epsilon>0$ a unique solution $\left(\check{\Gamma}^{\epsilon}, \hat{Z}^{\epsilon}, \check{Z}^{\epsilon}\right)$ of (5.2) with bounded $\check{\Gamma}^{\epsilon}$, and both $\int \hat{Z}^{\epsilon} \mathrm{d} W$ and $\int \check{Z}^{\epsilon} \mathrm{d} W^{\perp}$ are in $B M O(\mathbb{G}, P)$. As in the proof of Proposition 2.3, one can show that $\check{\Gamma}^{\epsilon} \leq \check{\Gamma}$ and that $\left|\check{\Gamma}^{\epsilon}\right|$ is bounded by $\|G\|_{L^{\infty}}+\frac{1}{2 \gamma}\left\|\int_{0}^{T}\left|\lambda_{s}\right|^{2} \mathrm{~d} s\right\|_{L^{\infty}}$, uniformly in $\epsilon$. Applying Itô's formula to $\exp \left(\check{\Gamma}^{\epsilon}\right)$ then yields like in the proof of Proposition 7 of Mania and Schweizer [13] that the $B M O(\mathbb{G}, P)$-norms of $\int \hat{Z}^{\epsilon} \mathrm{d} W$ and $\int \check{Z}^{\epsilon} \mathrm{d} W^{\perp}$ are bounded uniformly in $\epsilon$. By Theorem 3.6 of Kazamaki [11], the $B M O\left(\mathbb{G}, \check{P}^{\epsilon}\right)$-norm of $\int \hat{Z}^{\epsilon} \mathrm{d} W$ is thus bounded uniformly in $\epsilon$, where

$$
\frac{\mathrm{d} \check{P}^{\epsilon}}{\mathrm{d} P}:=\mathcal{E}\left(-\int \lambda \mathrm{d} W+\frac{\gamma}{2} \int\left(\check{Z}^{\epsilon}+\check{Z}\right) \mathrm{d} W^{\perp}\right)_{T} .
$$

We now obtain from (5.1) and (5.2) by conditioning on $\mathcal{G}_{t}$ under $\check{P}^{\epsilon}$ that

$$
0 \leq \check{\Gamma}_{t}-\check{\Gamma}_{t}^{\epsilon}=\epsilon E_{\check{P}^{\epsilon}}\left[\int_{t}^{T}\left|\hat{Z}_{s}^{\epsilon}\right|^{2} \mathrm{~d} s \mid \mathcal{G}_{t}\right] \leq \epsilon\left\|\int \hat{Z}^{\epsilon} \mathrm{d} W\right\|_{B M O_{2}\left(\mathbb{G}, \check{P}^{\epsilon}\right)}^{2},
$$

and this converges almost surely to 0 for $\epsilon \searrow 0$.

To apply the change of measure result in Theorem 3.1, we use notations analogous to Sect. 3.1, whose $B$ corresponds to $\left(W, W^{\perp}\right)$. Let us set

$$
\begin{aligned}
\gamma G_{t}^{\kappa, \epsilon} & :=\gamma G+\frac{1}{2} \int_{t}^{T}\left(\left|\lambda_{S}\right|^{2}+\epsilon^{-1}\left(\left|\lambda_{S}\right|^{2}-\left|\kappa_{S}\right|^{2}\right)\right) \mathrm{d} s-\epsilon^{-1} \int_{t}^{T}\left(\kappa_{S}-\lambda_{S}\right) \mathrm{d} W_{s}, \\
\frac{\mathrm{d} Q^{\kappa}}{\mathrm{d} P} & :=\mathcal{E}\left(-\int \kappa \mathrm{d} W\right)_{T} .
\end{aligned}
$$


Note that $\mathcal{K}=\mathcal{K}^{(m)}$ is here a set of $\mathbb{R}^{m}$-valued processes. The next result follows fairly directly from Lemma 5.2 and Theorem 3.1, but spelling out all details is rather tedious and gives no new insights; hence we only outline the argument. We apply Theorem 3.1 to (5.2) with $\tilde{\epsilon}:=\frac{\gamma \epsilon}{2}, \tilde{B}:=\left(W, W^{\perp}\right)^{\prime}, \tilde{n}:=m+n$,

$$
\tilde{\Lambda}:=\frac{1}{\gamma}\left(\begin{array}{cc}
\epsilon^{-1} I_{m \times m} & 0 \\
0 & I_{n \times n}
\end{array}\right), \tilde{\alpha}:=-\tilde{\Lambda}\left(\begin{array}{l}
\lambda \\
0
\end{array}\right) \text { and } \tilde{\chi}:=-\left(\frac{1}{2 \gamma \epsilon}+\frac{1}{2 \gamma}\right)|\lambda|^{2} .
$$

This gives $\delta_{t}^{\min }(\tilde{\Lambda})=1 / \gamma$, and now we obtain from Lemma 5.2 and (3.6) in Theorem 3.1 for $\tilde{\mathcal{K}}:=\mathcal{K}^{(m)} \times \mathcal{K}^{(n)}$ the following result.

Proposition 5.3 We have

$$
\check{\Gamma}_{t}=-\underset{\epsilon \in(0,1]}{\operatorname{ess} \inf } \operatorname{essinf} \operatorname{in}\left(\log E_{Q^{\kappa}}\left[\exp \left(-\gamma G_{t}^{\kappa, \epsilon}\right) \mid \mathcal{G}_{t}\right]^{1 / \gamma}\right.
$$

By picking arbitrary $\kappa \in \mathcal{K}^{(m)}$ and $\epsilon \in(0,1]$, the representation (5.3) allows us to get lower bounds for $\breve{\Gamma}_{t}$, and hence also for $V_{t}^{G}$ by Lemma 5.1. Note that $Q^{\kappa}$ is a martingale measure for $S$ only for $\kappa=\lambda$. In that case, $Q^{\kappa}$ equals the minimal martingale measure $\hat{P}$, and we get from (5.3) that

$$
\check{\Gamma}_{t} \geq-\log E_{\hat{P}}\left[\exp \left(-\gamma G-\frac{1}{2} \int_{t}^{T}\left|\lambda_{s}\right|^{2} \mathrm{~d} s\right) \mid \mathcal{G}_{t}\right]^{1 / \gamma} .
$$

\subsection{Projecting onto incompleteness}

This short section exploits the projection result from Sect. 3.2 to give an upper bound for $V_{0}^{G}$. For any process $Z$, we denote by $\mathbb{F}^{Z}=\left(\mathcal{F}_{s}^{Z}\right)_{0 \leq s \leq T}$ the $P$-augmented filtration generated by $Z$. In this section, $Z^{o}$ stands for the optional projection of $Z$ onto the filtration $\mathbb{F}^{W^{\perp}}$ under the minimal martingale measure $\hat{P}$, i.e., $Z_{\tau}^{o}=E_{\hat{P}}\left[Z_{\tau} \mid \mathcal{F}_{\tau}^{W^{\perp}}\right]$ for any $\mathbb{F}^{W^{\perp}}$-stopping time $\tau$.

Proposition 5.4 For any $s \in[0, T], V^{G}$ satisfies

$$
\left(\log \left(-V^{G}\right)\right)_{s}^{o} \geq \log E_{\hat{P}}\left[\exp \left(-E_{\hat{P}}\left[\gamma G \mid \mathcal{F}_{T}^{W^{\perp}}\right]-\frac{1}{2} \int_{s}^{T}\left(|\lambda|^{2}\right)_{r}^{o} \mathrm{~d} r\right) \mid \mathcal{F}_{s}^{W^{\perp}}\right]
$$

Proof Using (4.4), we can rewrite (5.1) in the form

$$
\check{\Gamma}_{s}=G-\int_{s}^{T}\left(\frac{\gamma}{2}\left|\check{Z}_{r}\right|^{2}-\frac{1}{2 \gamma}\left|\lambda_{r}\right|^{2}\right) \mathrm{d} r+\int_{S}^{T} \hat{Z}_{r} \mathrm{~d} \hat{W}_{r}+\int_{S}^{T} \check{Z}_{r} \mathrm{~d} W_{r}^{\perp} .
$$


By Remark 3.4, we have $\check{\Gamma}^{o} \leq \bar{\Gamma}$ where $(\bar{\Gamma}, \bar{Z})$ solves the BSDE

$$
\bar{\Gamma}_{s}=E_{\hat{P}}\left[G \mid \mathcal{F}_{T}^{W^{\perp}}\right]-\int_{s}^{T}\left(\frac{\gamma}{2}\left|\bar{Z}_{r}\right|^{2}-\frac{1}{2 \gamma}\left(|\lambda|^{2}\right)_{r}^{o}\right) \mathrm{d} r+\int_{s}^{T} \bar{Z}_{r} \mathrm{~d} W_{r}^{\perp}
$$

for $0 \leq s \leq T$. A direct calculation shows similarly to (2.4) that

$$
\bar{\Gamma}_{s}=-\frac{1}{\gamma} \log E_{\hat{P}}\left[\exp \left(-E_{\hat{P}}\left[\gamma G \mid \mathcal{F}_{T}^{W^{\perp}}\right]-\frac{1}{2} \int_{s}^{T}\left(|\lambda|^{2}\right)_{r}^{o} \mathrm{~d} r\right) \mid \mathcal{F}_{s}^{W^{\perp}}\right], \quad 0 \leq s \leq T,
$$

which concludes the proof since $V^{G}=-\exp (-\gamma \check{\Gamma})$ by Lemma 5.1.

Proposition 5.4 gives an upper bound for $V_{0}^{G}$ and thus also for $b_{0}$, but these bounds are rather rough. In the next section, we show how additional measurability assumptions can be exploited to derive other bounds via the symmetrisation result of Sect. 3.3.

\subsection{Symmetrising a nontradable claim}

Recall that $\mathbb{F}^{Z}=\left(\mathcal{F}_{s}^{Z}\right)_{0 \leq s \leq T}$ denotes the $P$-augmented filtration generated by a process $Z$. We recall the processes $Y$ and $W$ from (4.1) and write for brevity

$$
\mathbb{W}=\left(\mathcal{W}_{s}\right)_{0 \leq s \leq T} \text { for } \mathbb{F}^{W}, \quad \mathbb{Y}=\left(\mathcal{Y}_{s}\right)_{0 \leq s \leq T} \text { for } \mathbb{F}^{Y}, \quad \hat{\mathbb{Y}}=\left(\hat{\mathcal{Y}}_{s}\right)_{0 \leq s \leq T} \text { for } \mathbb{F}^{\hat{Y}}
$$

If $R \lambda$ is $\mathbb{Y}$-predictable, then $\hat{Y}$ from (4.4) is $\mathbb{Y}$-adapted and hence $\hat{Y} \subseteq \mathbb{Y}$. In general, however, none of the above three filtrations contains any other. We study two cases which were introduced by Frei and Schweizer [7] in a setting with one-dimensional $Y$ and $W$.

Cases We consider one of the following two situations:

(I) $G \in L^{\infty}\left(\mathcal{Y}_{T}, P\right), \lambda$ is $\mathbb{Y}$-predictable, and $R$ is $\mathbb{Y}$-predictable.

(II) $G \in L^{\infty}\left(\hat{\mathcal{Y}}_{T}, P\right), \lambda$ is $\mathbb{F}^{S, \hat{Y}}$-predictable, and $\lambda$ is $\mathbb{W}$-predictable.

Each case reflects a situation where the payoff $G$ is driven by $Y$ (or $\hat{Y}$ ), whereas hedging can only be done in $S$ which is imperfectly correlated with $Y$ (or $\hat{Y}$ ). Direct hedging in the underlying of $G$ may be impossible for two basic reasons: In case (I), its driver is not traded at all (e.g., a volatility or a consumer price index), whereas in case (II), it is traded in principle but not tradable for our investor, due to legal, liquidity, practicability, cost or other reasons. We refer to Section 4.1 of Frei and Schweizer [7] for a thorough explanation and motivation of the assumptions in cases (I) and (II).

We focus in this section on case (I) and first relate $V^{G}$ to a BSDE of the form (2.1). A similar result for case (II) is given in Proposition A.4 in the Appendix. Recall from (4.3) that $\Lambda:=\frac{1}{\gamma}\left(I-R R^{\prime}\right)^{-1}$. 
Proposition 5.5 In case (I), the BSDE

$$
\Gamma_{s}=G-\int_{s}^{T}\left(\frac{1}{2} Z_{r}^{\prime} \Lambda_{r}^{-1} Z_{r}-Z_{r}^{\prime} R_{r} \lambda_{r}-\frac{1}{2 \gamma}\left|\lambda_{r}\right|^{2}\right) \mathrm{d} r+\int_{s}^{T} Z_{r} \mathrm{~d} Y_{r}
$$

for $0 \leq s \leq T$ has a unique solution $(\Gamma, Z)$ where $\Gamma$ is a real-valued bounded continuous $(\mathbb{Y}, P)$-semimartingale and $Z$ is an $\mathbb{R}^{n}$-valued $\mathbb{Y}$-predictable process such that $\int_{0}^{T}\left|Z_{S}\right|^{2} \mathrm{~d} s<\infty$ almost surely. Moreover, $V^{G}=-\exp (-\gamma \Gamma)$.

Proposition 5.5 shows in particular that $V^{G}$ is $\mathbb{Y}$-adapted in case (I). This generalises Remark 3.3 of Ankirchner et al. [2] who made the same observation in a Markovian setting. It also shows that the distortion power $\delta^{\hat{B}}$ in Theorem 2 of Frei and Schweizer [7] can be chosen $\mathbb{Y}$-adapted.

Proof The BSDE (5.4) can be brought into the form (2.1) by defining

$$
B:=Y, \quad \mathbb{F}:=\mathbb{Y}, \quad \chi:=-\frac{1}{2} \lambda^{\prime}\left(\frac{1}{\gamma} I+R^{\prime} \Lambda R\right) \lambda \text { and } \alpha:=-\Lambda R \lambda,
$$

and so (5.4) has a unique solution $(\Gamma, Z)$ by Lemma 2.2. Using (4.1) then shows that $\left(\Gamma, R^{\prime} Z, \sqrt{I-R R^{\prime}} Z\right)$ solves (5.1), and

$$
E_{P}\left[\int_{0}^{T}\left(Z_{s}^{\prime} R_{s} R_{s}^{\prime} Z_{s}+Z_{s}^{\prime}\left(I-R_{s} R_{s}^{\prime}\right) Z_{s}\right) \mathrm{d} s\right]=E_{P}\left[\int_{0}^{T}\left|Z_{s}\right|^{2} \mathrm{~d} s\right]<\infty
$$

since $\int Z \mathrm{~d} Y \in B M O(\mathbb{Y}, P)$ by Lemma 2.2. Moreover, $V^{G}=-\exp (-\gamma \Gamma)$ by uniqueness for (5.1). For later use, note that plugging (5.5) into (3.2) gives

$$
\begin{aligned}
G_{t}^{\kappa}= & G+\frac{1}{2 \gamma} \int_{t}^{T}\left|\lambda_{S}\right|^{2} \mathrm{~d} s-\frac{1}{2} \int_{t}^{T}\left(\kappa_{s}^{\prime} \Lambda_{s} \kappa_{s}-\left(R_{S} \lambda_{s}\right)^{\prime} \Lambda_{S}\left(R_{S} \lambda_{s}\right)\right) \mathrm{d} s \\
& -\int_{t}^{T} \Lambda_{S}\left(\kappa_{s}-R_{S} \lambda_{S}\right) \mathrm{d} Y_{s} .
\end{aligned}
$$

The key point for rewriting the description of $V^{G}$ from (5.1) in Lemma 5.1 to (5.4) in Proposition 5.5 is that the latter BSDE has the form (2.1); and this reformulation, by working in the filtration $\mathbb{F}^{Y}$ instead of $\mathbb{G}=\mathbb{F}^{\left(W, W^{\perp}\right)}$, is possible thanks to the measurability conditions imposed by case (I). We could now apply to (5.4) all the results of Sect. 3, but we focus here on symmetrisation via Theorem 3.7. However, we also briefly mention in the next remarks some consequences of the probability change via Theorem 3.1 and the projection via Theorem 3.3. 
Remark 5.6 (1) Theorem 3.1 applied to the BSDE (5.4) generalises Theorem 2 of Frei and Schweizer [7], which corresponds to the choice $\kappa=R \lambda$. In that case, $G_{t}^{\kappa}$ from (5.6) simplifies to $G_{t}^{R \lambda}=G+\frac{1}{2 \gamma} \int_{t}^{T}\left|\lambda_{s}\right|^{2} \mathrm{~d} s$ and $P^{R \lambda}$ is the projection onto $\mathcal{Y}_{T}$ of the minimal martingale measure $\hat{P}$ in (4.5). The freedom in Theorem 3.1 of choosing $\kappa$ arbitrarily allows one to obtain other bounds. Note from (5.6) that $\kappa=R \lambda$ is special because only with this choice, $G_{t}^{\kappa}$ has no $\mathrm{d} Y$-integral in addition to $G$. So the minimality of $\hat{P}$ in the original sense corresponds to the minimality of $G_{t}^{R \lambda}$ in the sense that it only differs from $G$ by the terminal value of a finite variation process.

(2) Theorem 4 of Frei and Schweizer [8] is the general semimartingale analogue of Theorem 3.1 applied to (5.4), with slightly different assumptions.

(3) Proposition 5.5 starts with an optimisation problem in a financial market and relates this to the solution of a BSDE. In the opposite direction, one could also start with a BSDE and link its solution to an optimisation problem in an artificially constructed financial market. For the BSDE (2.1) with fixed $(G, \Lambda, \alpha, \chi)$ as in Sect. 2.1, we can define

$$
\begin{aligned}
& \gamma:=\sup _{s \in[t, T]}\left\|\max \operatorname{spec}\left(\Lambda_{s}^{-1}\right)\right\|_{L^{\infty}}+1, \quad R:=\sqrt{I-\frac{1}{\gamma} \Lambda^{-1}}, \quad \lambda:=-R^{-1} \Lambda^{-1} \alpha, \\
& \tilde{G}:=G+\int_{t}^{T} \chi_{s} \mathrm{~d} s+\frac{1}{2} \int_{t}^{T} \lambda_{s}^{\prime}\left(\frac{1}{\gamma} I+R_{s}^{\prime} \Lambda_{s} R_{s}\right) \lambda_{s} \mathrm{~d} s, \quad m:=n .
\end{aligned}
$$

If we construct with these parameters a model as in Sect. 4, then Proposition 5.5 yields $\Gamma_{t}=-\frac{1}{\gamma} \log \left(-V_{t}^{\tilde{G}}\right)$.

(4) Theorem 3.3 gives an upper bound for the solution of (5.4) in terms of a solution to a projected, lower-dimensional BSDE. Combining this with the above remark shows that projecting the optimisation problem relates to constructing a lower-dimensional artificial market.

Applying Theorem 3.1 to the BSDE (5.4) yields bounds for $V_{t}^{G}$ which depend directly on the claim $G$. If we also use symmetrisation via Theorem 3.7, we obtain bounds depending on a symmetrisation of $G$.

For any $\mathbb{Y}$-predictable $\mathcal{S}^{n}$-valued process $\Lambda$, there exist a $\mathbb{Y}$-predictable $O$ valued in $\mathrm{O}(n)$ and a $\mathbb{Y}$-predictable diagonal matrix $D=\operatorname{diag}\left(D^{11}, \ldots, D^{n n}\right)$ with $\Lambda=O^{\prime} D O$. For a bounded $\mathbb{Y}$-predictable process $\kappa$, we define a process $Y^{\kappa, O}$ null at 0 by $\mathrm{d} Y^{\kappa, O}=O(\mathrm{~d} Y+\kappa \mathrm{d} s)$, and we set $\mathbb{Y}^{\kappa, O}=\left(\mathcal{Y}_{s}^{\kappa, O}\right)_{0 \leq s \leq T}:=\mathbb{F}^{Y^{\kappa, O}}$. For the next result, we work on Wiener space with coordinate process $Y^{\kappa}, O$ and use the notations of Sects. 3.1 and 3.3 with $B:=Y^{\kappa, O}$ and $\chi, \alpha$ given by (5.5).

Proposition 5.7 Write $\Lambda=O^{\prime} D O$ and fix a bounded $\mathbb{Y}$-predictable process $\kappa$. In

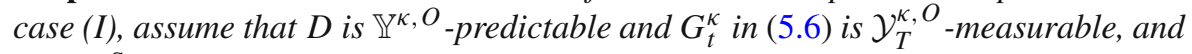
set $G_{t}^{\kappa, \text { Sym }}:=\frac{1}{n !} \sum_{u \in \operatorname{Perm}} G_{t}^{\kappa} \circ U_{t}$. Then we have 


$$
V_{t}^{G} \leq-E_{P^{\kappa}}\left[\exp \left(-G_{t}^{\kappa, \operatorname{Sym}} / d_{t}\right) \mid \mathcal{Y}_{t}^{\kappa, O}\right]^{\gamma d_{t}} \text { a.s. }
$$

where

$$
d_{t}:=\sup _{s \in[t, T]}\left\|\frac{1}{n} \sum_{j=1}^{n} \sup _{u \in \operatorname{Perm}}\left(D_{s}^{j j} \circ U_{t}\right)\right\|_{L^{\infty}} .
$$

Proof By Proposition 5.5, $V_{t}^{G}=-\exp \left(-\gamma \Gamma_{t}\right)$ where $(\Gamma, Z)$ solves (5.4). If $(\tilde{\Gamma}, \tilde{Z})$ solves for $0 \leq s \leq T$ the BSDE

$$
\tilde{\Gamma}_{s}=G_{t}^{\kappa}-\frac{1}{2} \int_{S}^{T}\left(O_{r} \tilde{Z}_{r}\right)^{\prime} D_{r}^{-1}\left(O_{r} \tilde{Z}_{r}\right) \mathrm{d} r+\int_{s}^{T}\left(O_{r} \tilde{Z}_{r}\right) \mathrm{d} Y_{r}^{\kappa, O}
$$

combining (5.8), (5.6) and (5.4) on $\llbracket 0, T \rrbracket$ shows $Z=\tilde{Z}-\Lambda(\kappa-R \lambda)$ and

$$
\Gamma=\tilde{\Gamma}-\frac{1}{2} \int_{t}^{t \vee}\left(|\lambda|^{2} / \gamma-(\kappa-R \lambda)^{\prime} \Lambda(\kappa+R \lambda)\right) \mathrm{d} s+\int_{t}^{t \vee} \Lambda(\kappa-R \lambda) \mathrm{d} Y
$$

so that in particular $\Gamma_{t}=\tilde{\Gamma}_{t}$. Now we apply Theorem 3.7 to the BSDE (5.8) and obtain (5.7) from (3.36), except for one detail: The payoff $G_{t}^{\kappa}$ from (5.6) is not bounded, as Theorem 3.7 requires. But a closer look shows that $G_{t}^{\kappa}$ differs from a bounded payoff only by $\int_{t}^{T} \Lambda_{s}\left(\kappa_{s}-R_{s} \lambda_{s}\right) \mathrm{d} Y_{s}$, and since this $Y$-integrand is bounded, the arguments from Theorem 3.7 still go through.

If we choose $\kappa=R \lambda$ in Proposition 5.7, the random variable $G_{t}^{\kappa}$ in (5.6) simplifies to $G_{t}^{R \lambda}=G+\frac{1}{2 \gamma} \int_{t}^{T}\left|\lambda_{s}\right|^{2} \mathrm{~d} s$ and the resulting upper bound

$$
V_{t}^{G} \leq-E_{\hat{P}}\left[\exp \left(-G_{t}^{R \lambda, \operatorname{Sym}} / d_{t}\right) \mid \mathcal{Y}_{t}^{R \lambda, O}\right]^{\gamma d_{t}} \text { a.s. }
$$

can be written under the minimal martingale measure $\hat{P}$ from (4.5). In general, the bound of Proposition 5.7 differs from the upper bound in Theorem 3.1 in two respects. On the one hand, the expectation in (5.7) is distorted by $d_{t}$ which depends on the average eigenvalue of (the permuted) $D$, whereas $\delta_{t}^{\max }$ from Theorem 3.1 reflects the maximal eigenvalue of $D$. We have $d_{t} \leq \delta_{t}^{\max }$ and in the multidimensional case $n>1$, there can be a big difference between $d_{t}$ and $\delta_{t}^{\max }$ so that the bound of Proposition 5.7 may significantly improve that of Theorem 3.1. But on the other hand, the bound of Proposition 5.7 depends on the symmetrised claim $G_{t}^{\kappa, \text { Sym }}$ instead of $G_{t}^{\kappa}$, which may make it worse. It depends on the concrete situation which of the two impacts is stronger and whether Proposition 5.7 or Theorem 3.1 gives the better bound. For $n=1$, the bounds coincide. 
In practice, the claim $G$ often has symmetry properties (e.g., if it is the sum of individual assets); then $G_{t}^{\kappa, \text { Sym }}$ does not differ much from $G_{t}^{\kappa}$, and the bounds of Proposition 5.7 can be much better than those from Theorem 3.1. We illustrate the above discussion in the next simple example.

Example Take $m=\operatorname{dim} W=1$ and $n=\operatorname{dim} Y=2$. We assume that instantaneous correlations between $W$ and $Y$ are given by $R=\left(\rho^{1}, \rho^{2}\right)^{\prime}$ for two constants $\left.\rho^{1}, \rho^{2} \in\right]-1,1\left[\right.$ with $0 \neq\left|\rho^{1}\right|^{2}+\left|\rho^{2}\right|^{2}<1$. By (4.3), we have

$$
\Lambda=\frac{1}{\gamma}\left(I-R R^{\prime}\right)^{-1}=\frac{1}{\gamma}\left(\begin{array}{cc}
1-\left|\rho^{1}\right|^{2} & -\rho^{1} \rho^{2} \\
-\rho^{1} \rho^{2} & 1-\left|\rho^{2}\right|^{2}
\end{array}\right)^{-1}
$$

which can be written as $\Lambda=O^{\prime} D O$ for

$$
D=\frac{1}{\gamma}\left(\begin{array}{cc}
1 & 0 \\
0 & \frac{1}{1-\left|\rho^{1}\right|^{2}-\left|\rho^{2}\right|^{2}}
\end{array}\right) \text { and } O=\frac{1}{\sqrt{\left|\rho^{1}\right|^{2}+\left|\rho^{2}\right|^{2}}}\left(\begin{array}{cc}
\rho^{2} & -\rho^{1} \\
\rho^{1} & \rho^{2}
\end{array}\right)
$$

We assume that $\lambda=\frac{\mu}{\sigma}$ is constant, and we consider a claim which is of the form $G=q^{1} Y_{T}^{1}+q^{2} Y_{T}^{2}=q^{\prime} Y_{T}$ for a constant $q=\left(q^{1}, q^{2}\right)^{\prime} \in \mathbb{R}^{2} \backslash\{0\}$. In this simple setting, $V^{G}$ can be explicitly determined. Indeed, writing $G=q^{\prime} Y_{t}+\int_{t}^{T} q \mathrm{~d} Y_{s}$, plugging this into (5.6) and choosing $\kappa=\Lambda^{-1} q+R \lambda$ leads to

$$
G_{t}^{\kappa}=q^{\prime} Y_{t}+\frac{1}{2}\left(\lambda^{2} / \gamma-2 \lambda R^{\prime} q-q^{\prime} \Lambda^{-1} q\right)(T-t)
$$

and because this is $\mathcal{Y}_{t}$-measurable, we get $V_{t}^{G}=-\exp \left(-\gamma G_{t}^{\kappa}\right)$. But note that this works only because $G$ is of the special form $G=\int_{0}^{T} q \mathrm{~d} Y$ and $q, R$ and $\lambda$ are deterministic.

Although $V_{t}^{G}$ is explicitly known here, we next also compare the bounds from Theorem 3.1 and Proposition 5.7 for the special choice $\kappa=R \lambda$. We choose this $\kappa$ since it does not depend on $G$ and also has nice consequences, as explained after Proposition 5.7; and we compute the bounds despite their non-optimality since they are explicit and illustrative. Applying Theorem 3.1 for $\kappa=R \lambda$ to the BSDE (5.4) gives with an easy computation for the indifference value in (4.7) the upper bound

$$
\begin{aligned}
b_{0} & \leq-\log E_{P R \lambda}\left[\exp \left(-G / \delta_{0}^{\max }\right)\right]^{\delta_{0}^{\max }} \\
& =-\frac{\gamma T}{2}\left(1-\left|\rho^{1}\right|^{2}-\left|\rho^{2}\right|^{2}\right)\left(\left|q^{1}\right|^{2}+\left|q^{2}\right|^{2}\right)-T \lambda\left(q^{1} \rho^{1}+q^{2} \rho^{2}\right),
\end{aligned}
$$

where $\delta_{0}^{\max }=\max \operatorname{spec}(\Lambda)=\frac{1}{\gamma\left(1-\left|\rho^{1}\right|^{2}-\left|\rho^{2}\right|^{2}\right)}$. For Proposition 5.7 with $\kappa=R \lambda$, we have to symmetrise with respect to $Y_{s}^{R \lambda, O}=O Y_{s}+O R \lambda s, 0 \leq s \leq T$. The symmetrised claim is 


$$
\begin{aligned}
G_{0}^{R \lambda, \text { Sym }} & =\frac{1}{2}\left(q^{1}, q^{2}\right) O^{\prime} Y_{T}^{R \lambda, O}+\frac{1}{2}\left(q^{1}, q^{2}\right) O^{\prime}\left(\begin{array}{c}
\left(Y_{T}^{R \lambda, O}\right)^{2} \\
\left(Y_{T}^{R \lambda, O}\right)^{1}
\end{array}\right)-T \lambda\left(q^{1} \rho^{1}+q^{2} \rho^{2}\right) \\
& =\frac{1}{2}\left(\tilde{q}^{1}+\tilde{q}^{2}\right)\left(\left(Y_{T}^{R \lambda, O}\right)^{1}+\left(Y_{T}^{R \lambda, O}\right)^{2}\right)-T \lambda\left(q^{1} \rho^{1}+q^{2} \rho^{2}\right),
\end{aligned}
$$

where

$$
\left(\begin{array}{c}
\tilde{q}^{1} \\
\tilde{q}^{2}
\end{array}\right):=O\left(\begin{array}{c}
q^{1} \\
q^{2}
\end{array}\right)=\frac{1}{\sqrt{\left|\rho^{1}\right|^{2}+\left|\rho^{2}\right|^{2}}}\left(\begin{array}{c}
\rho^{2} q^{1}-\rho^{1} q^{2} \\
\rho^{1} q^{1}+\rho^{2} q^{2}
\end{array}\right)
$$

and so Proposition 5.7 and (4.7) yield

$$
\begin{aligned}
b_{0} & \leq-\log E_{P R \lambda}\left[\exp \left(-G_{0}^{R \lambda, \mathrm{Sym}} / d_{0}\right)\right]^{d_{0}} \\
& =-\frac{\gamma T}{2}\left(1-\left|\rho^{1}\right|^{2}-\left|\rho^{2}\right|^{2}\right) \frac{\left(\tilde{q}^{1}+\tilde{q}^{2}\right)^{2}}{2-\left|\rho^{1}\right|^{2}-\left|\rho^{2}\right|^{2}}-T \lambda\left(q^{1} \rho^{1}+q^{2} \rho^{2}\right),
\end{aligned}
$$

where $d_{0}=\frac{1}{2} \operatorname{tr}(\Lambda)=\frac{1-\left|\rho^{1}\right|^{2} / 2-\left|\rho^{2}\right|^{2} / 2}{\gamma\left(1-\left|\rho^{1}\right|^{2}-\left|\rho^{2}\right|^{2}\right)}$. Due to the symmetry of the model, we can interchange $\rho^{1}$ and $\rho^{2}$ and, simultaneously, $q^{1}$ and $q^{2}$. This leads to

$$
\begin{aligned}
b_{0} \leq & -T \lambda\left(q^{1} \rho^{1}+q^{2} \rho^{2}\right)-\gamma T\left(1-\left|\rho^{1}\right|^{2}-\left|\rho^{2}\right|^{2}\right) \\
& \times \frac{\max \left\{\left(\rho^{1}\left(q^{1}-q^{2}\right)+\rho^{2}\left(q^{1}+q^{2}\right)\right)^{2},\left(\rho^{2}\left(q^{2}-q^{1}\right)+\rho^{1}\left(q^{1}+q^{2}\right)\right)^{2}\right\}}{2\left(2-\left|\rho^{1}\right|^{2}-\left|\rho^{2}\right|^{2}\right)\left(\left|\rho^{1}\right|^{2}+\left|\rho^{2}\right|^{2}\right)},
\end{aligned}
$$

which is a better bound for $b_{0}$ than (5.9) if and only if

$$
1<\frac{\max \left\{\left(\rho^{1}\left(q^{1}-q^{2}\right)+\rho^{2}\left(q^{1}+q^{2}\right)\right)^{2},\left(\rho^{2}\left(q^{2}-q^{1}\right)+\rho^{1}\left(q^{1}+q^{2}\right)\right)^{2}\right\}}{\left(\left|q^{1}\right|^{2}+\left|q^{2}\right|^{2}\right)\left(2-\left|\rho^{1}\right|^{2}-\left|\rho^{2}\right|^{2}\right)\left(\left|\rho^{1}\right|^{2}+\left|\rho^{2}\right|^{2}\right)} .
$$

We assume without loss of generality that $q^{1} \neq 0$. Then $q^{2}=c q^{1}$ for some $c \in \mathbb{R}$, and a calculation shows that (5.11) is equivalent to

$$
\left(\rho^{1}, \rho^{2}\right) \notin\left(\overline{\mathbb{D}}_{\frac{1}{\sqrt{2}}}\left(c^{-}, c^{+}\right) \cup \overline{\mathbb{D}}_{\frac{1}{\sqrt{2}}}\left(-c^{-},-c^{+}\right)\right) \cap\left(\overline{\mathbb{D}}_{\frac{1}{\sqrt{2}}}\left(c^{+},-c^{-}\right) \cup \overline{\mathbb{D}}_{\frac{1}{\sqrt{2}}}\left(-c^{+}, c^{-}\right)\right),
$$

where $c^{ \pm}:=\frac{1 \pm c}{2 \sqrt{1+|c|^{2}}}$ and $\overline{\mathbb{D}}_{\frac{1}{\sqrt{2}}}(z)$ denotes the closed disk of radius $1 / \sqrt{2}$ centered at $z \in \mathbb{R}^{2}$. Note that $\left|c^{-}\right|^{2}+\left|c^{+}\right|^{2}=1 / 2$ so that the centers of all four disks in (5.12) lie on a circle of radius $1 / \sqrt{2}$ centered at the origin. 

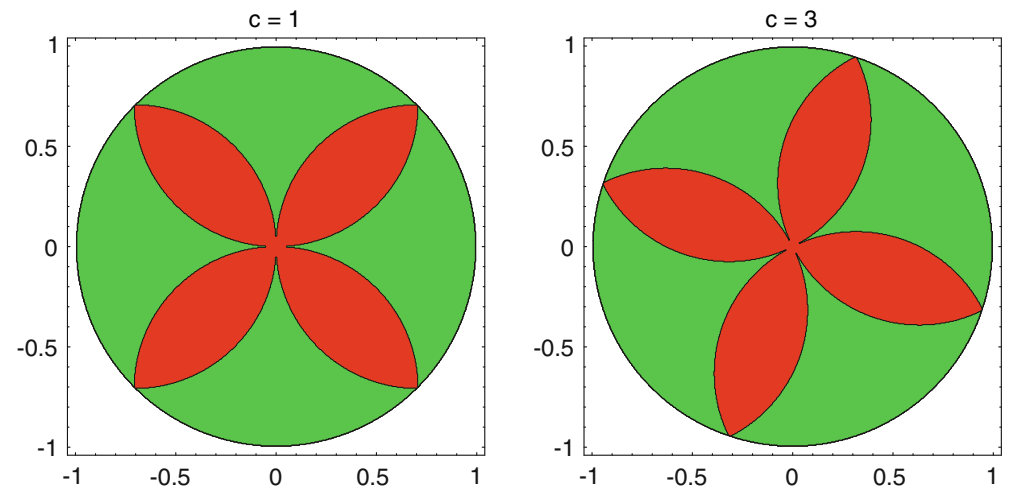

Fig. 1 Graphical visualisation of (5.12) for $c=1$ (left panel) and $c=3$ (right panel) with $\rho^{1}$ on the horizontal and $\rho^{2}$ on the vertical axis. We have $c^{-}=0, c^{+}=1 / \sqrt{2}$ (left panel) and $c^{-}=-1 / \sqrt{10}$, $c^{+}=2 / \sqrt{10}$ (right panel)

Figure 1 shows in green (light) the area on which (5.12) holds and in red (dark) its complement in the unit disk. In the green area, the symmetrised bound (5.10) is better than (5.9), and vice versa in the red area. The green area amounts to $2 / \pi \approx 63.66 \%$ of the total surface of the unit disk. In principle, the bigger $\left|\rho^{1}\right|^{2}+\left|\rho^{2}\right|^{2}$ is and the nearer $\left(\rho^{1}, \rho^{2}\right)$ is to one of the points $\left(c^{-}, c^{+}\right),\left(-c^{-},-c^{+}\right),\left(c^{+},-c^{-}\right)$or $\left(-c^{+}, c^{-}\right)$, the more likely it is that $\left(\rho^{1}, \rho^{2}\right)$ is in the green area and the symmetrised bound is better. This reflects the idea that if $G$ is more symmetric with respect to $Y^{R \lambda, O}$ and the eigenvalues of $\Lambda$ differ a lot, then making everything symmetric will achieve more than only squeezing the eigenvalues together.

Acknowledgments We thank Ying $\mathrm{Hu}$ and an anonymous referee for useful comments and questions that prompted us to improve some of our results. Financial support by the National Centre of Competence in Research "Financial Valuation and Risk Management" (NCCR FINRISK), Project D1 (Mathematical Methods in Financial Risk Management) is gratefully acknowledged. The NCCR FINRISK is a research instrument of the Swiss National Science Foundation.

\section{Appendix: Auxiliary results}

Lemma A.1 The function $f(A, z)=\frac{1}{2} z^{\prime} A^{-1} z$ in (2.2) is jointly convex.

Proof It is enough to show that, for fixed $z, y \in \mathbb{R}^{n}$ and $A, F \in \mathcal{S}^{n}$,

$$
z^{\prime} A^{-1} z+y^{\prime} F^{-1} y \geq(z+y)^{\prime}(A+F)^{-1}(z+y) .
$$

We first note that there is $C \in \operatorname{GL}(n)$ such that $C^{\prime} A C=I$ and $D:=C^{\prime} F C$ is diagonal. Indeed, $A=U^{\prime} U$ for some $U \in \mathrm{GL}(n)$, and $\left(U^{-1}\right)^{\prime} F U^{-1}$ is symmetric; so there exists $V \in \mathrm{O}(n)$ with $V^{\prime}\left(U^{-1}\right)^{\prime} F U^{-1} V$ diagonal, and $C:=U^{-1} V$ will do. Thus (A.1) is equivalent to

$$
|\check{z}|^{2}+\check{y}^{\prime} D^{-1} \check{y} \geq(\check{z}+\check{y})^{\prime}(I+D)^{-1}(\check{z}+\check{y}), \quad \check{z}:=C^{\prime} z \text { and } \check{y}:=C^{\prime} y \text {, }
$$


or, with $D=\operatorname{diag}\left(D^{11}, \ldots, D^{n n}\right)$, to

$$
\sum_{j=1}^{n}\left(\left|\check{z}^{j}\right|^{2}+\left|\check{y}^{j}\right|^{2} / D^{j j}\right) \geq \sum_{j=1}^{n} \frac{\left|\check{z}^{j}+\check{y}^{j}\right|^{2}}{1+D^{j j}}
$$

But the last relation is true because for $j=1, \ldots, n$, we have

$$
\left(\left|\check{z}^{j}\right|^{2}+\left|\check{y}^{j}\right|^{2} / D^{j j}\right)-\frac{\left|\check{z}^{j}+\check{y}^{j}\right|^{2}}{1+D^{j j}}=\frac{\left|\check{z}^{j} \sqrt{D^{j j}}-\check{y}^{j} / \sqrt{D^{j j}}\right|^{2}}{1+D^{j j}} \geq 0 .
$$

Lemma A.2 In the setting of Sect. 3.1, fix $\delta>0$. If a predictable $\mathbb{R}^{n}$-valued process $\kappa$ with $\int \kappa \mathrm{d} B$ in $B M O(P)$ satisfies

$$
\left\|\int \kappa \mathrm{d} B\right\|_{B M O_{2}(P)}^{2}<\frac{1}{3\left|\delta_{t}^{\max } / \delta\right|^{2}+3 \delta_{t}^{\max } / \delta+1},
$$

then $\kappa$ is in $\mathcal{K}^{\delta}$.

Proof We show that $\kappa$ satisfies (3.3). For $p>1$ to be specified later and for any stopping time $\tau$ valued in $[t, T]$, we have

$$
\begin{gathered}
E_{P^{\kappa}}\left[\exp \left(\int_{\tau}^{T} \frac{1}{2} \kappa_{S}^{\prime} \Lambda_{s} \kappa_{S} \mathrm{~d} s+\int_{\tau}^{T} \Lambda_{s} \kappa_{S} \mathrm{~d} B_{S}\right)^{p / \delta} \mid \mathcal{F}_{\tau}\right] \\
=E_{P}\left[\left|\frac{\mathcal{E}\left(2 \int\left(\frac{p}{\delta} \Lambda-I\right) \kappa \mathrm{d} B\right)_{T}}{\mathcal{E}\left(2 \int\left(\frac{p}{\delta} \Lambda-I\right) \kappa \mathrm{d} B\right)_{\tau}}\right|^{1 / 2}\right. \\
\left.\times \exp \left(\int_{\tau}^{T} \kappa_{s}^{\prime}\left(\frac{p^{2}}{\delta^{2}} \Lambda_{s}^{2}-\frac{3 p}{2 \delta} \Lambda_{s}+\frac{1}{2} I\right) \kappa_{S} \mathrm{~d} s\right) \mid \mathcal{F}_{\tau}\right] \\
\leq E_{P}\left[\exp \left(\int_{\tau}^{T} 2 \kappa_{s}^{\prime}\left(\frac{p^{2}}{\delta^{2}} \Lambda_{s}^{2}-\frac{3 p}{2 \delta} \Lambda_{S}+\frac{1}{2} I\right) \kappa_{S} \mathrm{~d} s\right) \mid \mathcal{F}_{\tau}\right]^{1 / 2}
\end{gathered}
$$

by the Cauchy-Schwarz inequality. The John-Nirenberg inequality (Theorem 2.2 of Kazamaki [11]) implies that this is bounded uniformly in $\tau$ if

$$
\left(\frac{2 p^{2}}{\delta^{2}}\left|\delta_{t}^{\max }\right|^{2}+1\right)\left\|\int \kappa \mathrm{d} B\right\|_{B M O_{2}(P)}^{2}<1
$$


which is satisfied for the choice $p=\sqrt{3 / 2}$ under the assumption (A.2). Using additionally Jensen's inequality, we obtain analogously that

$$
\begin{aligned}
& E_{P^{\kappa}} {\left[\exp \left(\int_{\tau}^{T} \frac{1}{2} \kappa_{S}^{\prime} \Lambda_{s} \kappa_{S} \mathrm{~d} s+\int_{\tau}^{T} \Lambda_{S} \kappa_{s} \mathrm{~d} B_{S}\right)^{1 / \delta} \mid \mathcal{F}_{\tau}\right] } \\
& \geq\left|E_{P^{\kappa}}\left[\exp \left(-\int_{\tau}^{T} \frac{1}{2} \kappa_{S}^{\prime} \Lambda_{s} \kappa_{S} \mathrm{~d} s-\int_{\tau}^{T} \Lambda_{s} \kappa_{S} \mathrm{~d} B_{S}\right)^{1 / \delta} \mid \mathcal{F}_{\tau}\right]\right|^{-1}
\end{aligned}
$$

is bounded away from zero uniformly in $\tau$ if

$$
\left(\frac{2}{\delta^{2}}\left|\delta_{t}^{\max }\right|^{2}+\frac{3}{\delta} \delta_{t}^{\max }+1\right)\left\|\int \kappa \mathrm{d} B\right\|_{B M O_{2}(P)}^{2}<1,
$$

which is again fulfilled under the assumption (A.2). This implies that, after a division by $\exp \left(\int_{t}^{\tau} \frac{1}{2} \kappa_{s}^{\prime} \Lambda_{s} \kappa_{S} \mathrm{~d} s+\int_{t}^{\tau} \Lambda_{s} \kappa_{S} \mathrm{~d} B_{S}\right)^{p / \delta}$, the conditional expectation on the right-hand side of (3.3) is bounded away from zero, and the left-hand side of (3.3) is bounded. Therefore, there exists a constant $C$ such that (3.3) holds, which concludes the proof.

Lemma A.3 In the setting of Sect. 3.3, we have

$$
\left(\int Z \mathrm{~d} B\right) \circ U_{t}=\int\left(Z \circ U_{t}\right) \mathrm{d} B^{u} .
$$

for any predictable process $Z$ on Wiener space with $\int_{0}^{T}\left|Z_{s}\right|^{2} \mathrm{~d} s<\infty$ a.s.

Proof By Itô's representation theorem, any local martingale is of the form $c+\int \beta \mathrm{d} B$ for a constant $c$ and a predictable process $\beta$ with $\int_{0}^{T}\left|\beta_{s}\right|^{2} \mathrm{~d} s<\infty$ a.s. Therefore, (A.3) is equivalent to

$$
\left\langle\left(\int Z \mathrm{~d} B\right) \circ U_{t}, \int \beta \mathrm{d} B\right\rangle=\left\langle\int\left(Z \circ U_{t}\right) \mathrm{d} B^{u}, \int \beta \mathrm{d} B\right\rangle
$$

for any predictable $\beta$ with $\int_{0}^{T}\left|\beta_{s}\right|^{2} \mathrm{~d} s<\infty$ a.s. To prove (A.4), we note first that $P \circ U_{t}^{-1}=P$ by the invariance of Wiener measure under orthogonal transformations, and thus

$$
E\left[X \circ U_{t}\right]=E[X] \text { for all } X \in L^{1} \text {. }
$$

This implies that the (local) martingale property is invariant under $U_{t}$, i.e., for an adapted integrable process $M$, we have

$M$ is a (local) martingale $\Longleftrightarrow M \circ U_{t}$ is a (local) martingale. 
Indeed, if $\tau$ is a stopping time and $M_{\tau \wedge}$. is a martingale, then $\tau \circ U_{t}$ is a stopping time and we have for any $s \in[0, T]$ and $A \in \mathcal{F}_{S}$ that

$$
\begin{aligned}
E\left[\left(M \circ U_{t}\right)_{\left(\tau \circ U_{t}\right) \wedge T} \mathbb{1}_{A}\right] & =E\left[\left(M_{\tau \wedge T} \mathbb{1}_{U_{t}^{-1}(A)}\right) \circ U_{t}\right]=E\left[M_{\tau \wedge T} \mathbb{1}_{U_{t}^{-1}(A)}\right] \\
& =E\left[M_{\tau \wedge s} \mathbb{1}_{U_{t}^{-1}(A)}\right]=E\left[\left(M \circ U_{t}\right)_{\tau \wedge s} \mathbb{1}_{A}\right]
\end{aligned}
$$

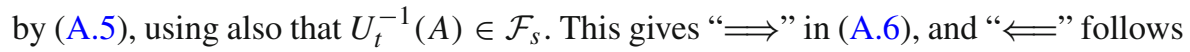
by symmetry.

We are now ready to prove (A.4). Its left-hand side equals

$$
\left\langle\left(\int Z \mathrm{~d} B\right) \circ U_{t}, \int \beta \mathrm{d} B\right\rangle=\int \mathrm{d}\left\langle\left(\int Z \mathrm{~d} B\right) \circ U_{t}, B\right\rangle \beta,
$$

and by (A.6) we have

$$
\begin{aligned}
\left\langle\left(\int Z \mathrm{~d} B\right) \circ U_{t}, B\right\rangle & =\left\langle\left(\int Z \mathrm{~d} B\right) \circ U_{t},\left(\left(\mathbb{1}_{\llbracket 0, t \rrbracket} I+\mathbb{1}_{\rrbracket t, T \rrbracket} u^{-1}\right) B\right) \circ U_{t}\right\rangle \\
& =\left\langle\int Z \mathrm{~d} B,\left(\mathbb{1}_{\llbracket 0, t \rrbracket} I+\mathbb{1}_{\rrbracket t, T \rrbracket} u^{-1}\right) B\right\rangle \circ U_{t} \\
& =\int_{0}^{t \wedge} Z^{\prime} \mathrm{d} s+\left(\int_{t}^{t \vee} Z^{\prime}\left(u^{-1}\right)^{\prime} \mathrm{d} s\right) \circ U_{t} .
\end{aligned}
$$

Since $\left(\int Z^{\prime}\left(u^{-1}\right)^{\prime} \mathrm{d} s\right) \circ U_{t}=\int\left(Z \circ U_{t}\right)^{\prime} u \mathrm{~d} s$, we obtain from (A.7) that

$$
\begin{aligned}
\left\langle\left(\int Z \mathrm{~d} B\right) \circ U_{t}, \int \beta \mathrm{d} B\right\rangle & =\int\left(\mathbb{1}_{\llbracket 0, t \rrbracket} Z^{\prime} \beta+\mathbb{1}_{\rrbracket t, T \rrbracket}\left(Z \circ U_{t}\right)^{\prime} u \beta\right) \mathrm{d} s \\
& =\left\langle\int\left(Z \circ U_{t}\right) \mathrm{d} B^{u}, \int \beta \mathrm{d} B\right\rangle
\end{aligned}
$$

which shows (A.4) and concludes the proof.

The following result is the analogue of Proposition 5.5 for case (II).

Proposition A.4 Under the assumptions of case (II) in Sect. 5.3 with the additional requirement that $R$ is $\hat{\mathbb{Y}}$-predictable, the $B S D E$

$$
\Gamma_{s}=G-\int_{s}^{T} \frac{1}{2} Z_{r}^{\prime} \Lambda_{r}^{-1} Z_{r} \mathrm{~d} r+\int_{s}^{T} Z_{r} \mathrm{~d} \hat{Y}_{r}, \quad 0 \leq s \leq T
$$

has a unique solution $(\Gamma, Z)$ where $\Gamma$ is a real-valued bounded continuous $(\hat{\mathbb{Y}}, \hat{P})$-semimartingale and $Z$ is an $\mathbb{R}^{n}$-valued $\hat{\mathbb{Y}}$-predictable process such that $\int_{0}^{T}\left|Z_{S}\right|^{2} \mathrm{~d} s<\infty$ almost surely. Moreover, for any $s \in[0, T]$, 


$$
V_{s}^{G}=-\exp \left(-\gamma \Gamma_{s}-\frac{1}{2} E_{\hat{P}}\left[\int_{s}^{T}\left|\lambda_{r}\right|^{2} \mathrm{~d} r \mid \mathcal{W}_{s}\right]\right) \text { a.s. }
$$

Proof This follows the same idea as Proposition 5.5, using additionally that the meanvariance tradeoff $\int_{0}^{T}\left|\lambda_{r}\right|^{2} \mathrm{~d} r$ is in case (II) attainable by trading in $S$. In more detail, we replace $P$ in Sect. 2 by $\hat{P}$ and set

$$
B:=\hat{Y}, \quad \mathbb{F}:=\hat{\mathbb{Y}}, \quad \alpha:=0 \text { and } \chi:=0
$$

to bring (A.8) into the form (2.1). By Lemma 2.2, (A.8) has a unique solution $(\Gamma, Z)$, and $\int Z \mathrm{~d} \hat{Y}$ is in $B M O(\hat{\mathbb{Y}}, \hat{P})$. Since $R \lambda$ is bounded, $\int Z \mathrm{~d} Y$ is a $(\mathbb{G}, P)$-martingale, and because $\Gamma$ is bounded, we obtain from (A.8) that $E_{P}\left[\frac{1}{2} \int_{0}^{T} Z_{s}^{\prime} \Lambda_{s}^{-1} Z_{s} \mathrm{~d} s\right]<\infty$, which implies $E_{P}\left[\int_{0}^{T}\left|Z_{s}\right|^{2} \mathrm{~d} s\right]<\infty$ due to (4.2). To deal with the term involving $\lambda$, we use Itô's representation theorem as in Lemma 1.6.7 of Karatzas and Shreve [10] and obtain a $\mathbb{W}$-predictable process $\eta=\left(\eta_{s}\right)_{0 \leq s \leq T}$ with $E_{\hat{P}}\left[\int_{0}^{T}\left|\eta_{s}\right|^{2} \mathrm{~d} s\right]<\infty$ and

$$
\begin{aligned}
\frac{1}{2 \gamma} \int_{0}^{T}\left|\lambda_{s}\right|^{2} \mathrm{~d} s & =\frac{1}{2 \gamma} E_{\hat{P}}\left[\int_{0}^{T}\left|\lambda_{s}\right|^{2} \mathrm{~d} s\right]+\int_{0}^{T} \eta_{s} \mathrm{~d} \hat{W}_{s} \\
& =\frac{1}{2 \gamma} E_{\hat{P}}\left[\int_{0}^{T}\left|\lambda_{s}\right|^{2} \mathrm{~d} s\right]+\int_{0}^{T} \eta_{s}^{\prime} \lambda_{s} \mathrm{~d} s+\int_{0}^{T} \eta_{s} \mathrm{~d} W_{s} .
\end{aligned}
$$

Here we use that $\lambda$ is $\mathbb{W}$-predictable in case (II), recalling that $\mathbb{W}=\mathbb{F}^{W}$. As $\lambda$ is bounded, $\int \eta \mathrm{d} \hat{W}$ is in $B M O(\mathbb{G}, \hat{P})$ and so $\int \eta \mathrm{d} W$ is in $B M O(\mathbb{G}, P)$ by Theorem 3.6 of Kazamaki [11]. For the solution $(\Gamma, Z)$ of $(\mathrm{A} .8)$, we set

$$
(\check{\Gamma}, \hat{Z}, \check{Z}):=\left(\Gamma+\frac{1}{2 \gamma} E_{\hat{P}}\left[\int\left|\lambda_{s}\right|^{2} \mathrm{~d} s \mid \mathcal{W} .\right],-\eta+R^{\prime} Z, \sqrt{I-R R^{\prime}} Z\right)
$$

and calculate

$$
\begin{aligned}
\mathrm{d} \check{\Gamma}_{s} & =\mathrm{d} \Gamma_{s}+\frac{1}{2 \gamma} \mathrm{d}\left(E_{\hat{P}}\left[\int_{0}^{T}\left|\lambda_{r}\right|^{2} \mathrm{~d} r \mid \mathcal{W}_{s}\right]-\int_{0}^{s}\left|\lambda_{r}\right|^{2} \mathrm{~d} r\right) \\
& =\mathrm{d} \Gamma_{s}+\eta_{s}^{\prime} \lambda_{s} \mathrm{~d} s+\eta_{s} \mathrm{~d} W_{s}-\frac{1}{2 \gamma}\left|\lambda_{s}\right|^{2} \mathrm{~d} s \\
& =\left(\frac{\gamma}{2}\left|\check{Z}_{s}\right|^{2}-\hat{Z}_{s}^{\prime} \lambda_{s}-\frac{1}{2 \gamma}\left|\lambda_{s}\right|^{2}\right) \mathrm{d} s-\hat{Z}_{s} \mathrm{~d} W_{s}-\check{Z}_{s} \mathrm{~d} W_{s}^{\perp}, \quad 0 \leq s \leq T
\end{aligned}
$$


by (4.1), (4.4) and (A.10). Therefore, $(\check{\Gamma}, \hat{Z}, \check{Z})$ solves (5.1) and we also have

$$
\begin{gathered}
E_{P}\left[\int_{0}^{T}\left(\left(\eta_{s}+R_{s}^{\prime} Z_{S}\right)^{\prime}\left(\eta_{s}+R_{s}^{\prime} Z_{s}\right)+Z_{s}^{\prime}\left(I-R_{s} R_{s}^{\prime}\right) Z_{s}\right) \mathrm{d} s\right] \\
\leq 3 E_{P}\left[\int_{0}^{T}\left|Z_{s}\right|^{2} \mathrm{~d} s\right]+2 E_{P}\left[\int_{0}^{T}\left|\eta_{s}\right|^{2} \mathrm{~d} s\right]<\infty .
\end{gathered}
$$

Finally, (A.9) follows from the uniqueness of solutions to (5.1).

\section{References}

1. Alvino, A., Lions, P.-L., Trombetti, G.: Comparison results for elliptic and parabolic equations via symmetrization: a new approach. Differ. Integral Equ. 4, 25-50 (1991)

2. Ankirchner, S., Imkeller, P., Reis, G.: Pricing and hedging of derivatives based on non-tradable underlyings. Math. Finance (to appear). http://wahrscheinlichkeitstheorie.hu-berlin.de/

3. Azoff, E.A.: Borel measurability in linear algebra. Proc. Am. Math. Soc. 42, 346-350 (1974)

4. Barrieu, P., El Karoui, N.: Pricing, hedging, and designing derivatives with risk measures. In: Carmona, R. (ed.) Indifference Pricing: Theory and Applications, pp. 77-146. Princeton University Press (2009)

5. Briand, P., Hu, Y.: Quadratic BSDEs with convex generators and unbounded terminal conditions. Probab. Theory Relat. Fields 141, 543-567 (2008)

6. El Karoui, N., Peng, S., Quenez, M.C.: Backward stochastic differential equations in finance. Math. Finance 7, 1-71 (1997)

7. Frei, C., Schweizer, M.: Exponential utility indifference valuation in two Brownian settings with stochastic correlation. Adv. Appl. Probab. 40, 401-423 (2008)

8. Frei, C., Schweizer, M.: Exponential utility indifference valuation in a general semimartingale model. In: Delbaen, F., Rásonyi, M., Stricker, C. (eds.) Optimality and Risk-Modern Trends in Mathematical Finance. The Kabanov Festschrift, pp. 49-86. Springer, Berlin (2009)

9. Hu, Y., Imkeller, P., Müller, M.: Utility maximization in incomplete markets. Ann. Appl. Probab. 15, 1691-1712 (2005)

10. Karatzas, I., Shreve, S.: Methods of Mathematical Finance. Applications of Mathematics, vol. 39. Springer, New York (1998)

11. Kazamaki, N.: Continuous Exponential Martingales and $B M O$. Lecture Notes in Mathematics, vol. 1579. Springer, New York (1994)

12. Kobylanski, M.: Stochastic differential equations and partial differential equations with quadratic growth. Ann. Probab. 28, 558-602 (2000)

13. Mania, M., Schweizer, M.: Dynamic exponential utility indifference valuation. Ann. Appl. Probab. 15, 2113-2143 (2005) 